\title{
Pacific
}

Journal of

Mathematics

\section{DETERMINING MULTIPLICITIES OF HALF-INTEGRAL WEIGHT NEWFORMS}

Thomas RichaRd Shemanske AND Lynne WALLING 


\title{
DETERMINING MULTIPLICITIES OF HALF-INTEGRAL WEIGHT NEWFORMS
}

\author{
Thomas R. Shemanske and Lynne H. WALling
}

We decompose the space of newforms of half-integral weight into a direct sum of spaces of newforms of integral weight which occur with multiplicity one or two. This not only demonstrates in a precise way the failure of a multiplicity-one result to hold for half-integral weight newforms, but moreover indicates which spaces occur with a given multiplicity. The spaces occuring with multiplicity two are shown to be in one-to-one correspondence with a collection of Kohnen subspaces. As a consequence, it is shown that under the Shimura correspondence, the level a newform of half-integral weight is not determined by the level of the integral weight newform to which it corresponds.

Since a knowledge of the Hecke eigenvalues is insufficient to charectesize half-integral weight newforms up to a scalar, we develop sufficient conditions on the squarefree coefficients, augmenting the information on the eigenvalues, which allow such a characterization. In the last section, these later results are carried over to the Hilbert modular setting.

Introduction. It is well-known that newforms of half-integral weight do not satisfy a multiplicity-one theorem, that is in general, they cannot be characterized solely by their Hecke eigenvalues $([5])$. In one attempt to deal with this difficulty, Kohnen [6] defines a subspace of the space of half-integral weight cusp forms which does have a newform theory in which the newforms satisfy a multiplicityone theorem. On the other hand, he develops this theory only for cusp forms having restricted Fourier expansions as well as squarefree level and quadratic character.

In $[\mathbf{1 6}, \mathbf{1 7}]$, Ueda significantly extends Kohnen's theory by establishing trace identities which relate the trace of Hecke operators on various spaces of cusp forms of integral and half-integral weight. By using these trace identities, Ueda is able to give a decomposition 
of the Kohnen subspace for general levels as a direct sum of spaces of newforms of integral weight. Using similar techniques, one could also use Ueda's theorem to get a decomposition of the full space of cusp forms of half-integral weight.

In this paper we go in a somewhat different direction. We consider the subspace of half-integral weight newforms, but in the full space of cusp forms and not in the Kohnen subspace. We first use Ueda's theorem to obtain precise information about the failure of multiplicity-one by explicitly decomposing certain spaces of halfintegral weight newforms (as modules for appropriate Hecke algebras) in such a way as to demonstrate that the multiplicities which occur are either one or two, and by identifying those spaces which occur with a given multiplicity. We also observe that the spaces which occur with multiplicity two are in one-to-one correspondence with a certain (complete) collection of Kohnen subspaces.

Given that the Hecke operators commute with the Shimura lift, one is naturally led to consider all cusp forms of a given level and character which have the same eigenvalues for almost all the Hecke operators as a given integral weight newform $F$, typically denoted $S_{k / 2}(4 N, \psi, F)$. We show that, in contrast to possible expectations, the levels $N$ for which the subspace of newforms $S_{k / 2}^{+}(4 N, \psi, F) \neq$ $\{0\}$ are not determined by the level of $F$; in fact we show the existence of half-integral weight newforms of arbitrarily high level which have the same Hecke eigenvalues as $F$.

We also show that the Kohnen subspace of newforms is contained in the full space of newforms whenever the Kohnen space is defined. This is not obvious since the space of oldforms for the Kohnen subspace and the space of oldforms for the full space of cusp forms are given by different definitions, and the spaces of newforms are defined relative to the subspaces generated by the oldforms.

Finally we turn to the problem of determining sufficient conditions to characterize up to constant multiple newforms in the full space of cusp forms with arbitrary level and character. Let $N$ be a positive integer, $\psi$ an even Dirichlet character defined modulo $4 N$, and let $f \in S_{k / 2}(4 N, \psi)$ be a cusp form of weight $k / 2$, level $4 N$, and character $\psi$ with Fourier expansion $f=\sum a(n) q^{n}$. An immediate problem which one confronts is that unlike the integral weight case, a newform of half-integeral weight need not be a simultaneous eigen- 
form for all the Hecke operators. However, if $f$ is a simultaneous eigenform for all of the Hecke operators $T_{p^{2}}$, then a knowledge of the Hecke eigenvalues for these operators is sufficient to characterize the Fourier coefficients $a\left(t n^{2}\right)$ for any integer $n$ given the value of $a(t)$ for a squarefree positive integer $t$. It is therefore completely elementary to see that if $f$ is a simultaneous eigenform for all the Hecke operators $T_{p^{2}}$ and all of the coefficients $a(t)$ for squarefree $t$ are known, then $f$ is characterized completely. So the question is, how much can we weaken these assumptions and still characterize a cusp form $f$ ?

In a remarkable work, Waldspurger [20] showed (under mild restrictions) that for $f=\sum a(n) q^{n} \in S_{k / 2}(4 N, \psi, F)$, the value of $a(t)^{2}$ ( $t$ squarefree) is proportional to the "central" value of twists of the $L$-series attached to the integral weight newform $F$. However, the sign of the coefficient is undetermined. Thus while the absolute value of the coefficient is known, the actual value is not, and by a theorem of Vigneras, we know that (except for two exceptional cases) there are always an infinite number of squarefree integers $t$ for which the sign of the $a\left(t n^{2}\right)$ must be determined. Our results represent a first attempt at dealing with this uncertainty.

We show that if $f$ is a simultaneous eigenform (not necessarily a newform) for all of the Hecke operators $T_{p^{2}}$ and the coefficients $a(t)$ are known for all but a finite number of squarefree positive integers $t$, then $f$ is characterized completely. This is a straightforward consequence of a result of Vigneras. Next, we assume that $f$ is newform and require a knowledge of the values of the coefficients $a(t)$ for all but a finite number of squarefree positive integers $t$, but assume only that $f$ is a simultaneous eigenform for all but a finite number of the Hecke operators $T_{p^{2}}$. Once again, we can characterize $f$, but the proof requires a good deal more work, including an investigation of a half-integral weight analog of the Atkin-Lehner involution $W_{q}$. In the final section of the paper we carry over these last results to the Hilbert modular case.

1. Preliminaries. In general, the notation will be as in [14], but for convenience we remind the reader of few conventions. We let $\mathcal{G}$ denote the group extension of $\mathrm{GL}_{2}^{+}(\mathbb{R})$ consisting of pairs $[A, \varphi(\tau)]$ where $A=\left(\begin{array}{ll}a & b \\ c & d\end{array}\right) \in \mathrm{GL}_{2}^{+}(\mathbb{R})$ and where $\varphi$ is a holo- 
morphic function on the upper half-plane $\mathcal{H}$ satisfying $\varphi^{2}(\tau)=$ $t(a d-b c)^{-1 / 2}(c \tau+d)$ with $|t|=1$. We refer to the function $\varphi$ as a factor of automorphy for the matrix $A$. The group law in $\mathcal{G}$ is given by $[A, \varphi(\tau)][B, \psi(\tau)]=[A B, \varphi(B \tau) \psi(\tau)]$. For $A=$ $\left(\begin{array}{ll}a & b \\ c & d\end{array}\right) \in \Gamma_{0}(4)$ and $\theta(\tau)=\sum_{n \in \mathbb{Z}} e^{2 \pi i n^{2} \tau}, j(A, \tau)=\theta(A \tau) / \theta(\tau)$ is a well-defined factor of automorphy; we put $A^{*}=[A, j(A, \tau)] \in \mathcal{G}$. For any subgroup $\Gamma \subset \Gamma_{0}(4)$, we define a subgroup $\Gamma^{*}$ of $\mathcal{G}$ by $\Gamma^{*}=\left\{A^{*} \mid A \in \Gamma\right\}$. We shall also have occasion to use the group $\Gamma_{0}(N, M)=\left\{A=\left(\begin{array}{ll}a & b \\ c & d\end{array}\right) \in \Gamma_{0}(N) \mid b \equiv 0(\bmod M)\right\}$.

For $M$ a positive integer, $\psi$ a Dirichlet character defined modulo $M$, and $\ell$ a positive integer or half-integer, we let $S_{\ell}(M, \psi)$ denote the space of cusp forms of weight $\ell$ for $\Gamma_{0}(M)$ with character $\psi$ (see [7], [14]). For half-integral weight forms we will always assume that $M$ is divisible by 4 . We make one exception to this notation: we let $S_{3 / 2}(4 N, \psi)$ denote the orthogonal complement (in the full space of cusp forms) of the space generated by the theta series $h_{\psi}$ (see [14]). Henceforth let $N$ be a positive integer, $\psi$ a Dirichlet character defined modulo $4 N$ and $k=2 \lambda+1 \geq 3$ a positive odd integer.

For a prime $p$, the Hecke operators $T_{p^{2}}$ are defined as in [14]. For $f \in S_{k / 2}(4 N, \psi)$ and $q$ a prime dividing $N$, we define the Hecke operator (see [4]) $T_{q}$ by

$$
f\left|T_{q}=q^{\frac{k}{4}-1} f\right| \xi \mid \sum_{j} A_{j}^{*}
$$

where $\xi=\left[\left(\begin{array}{ll}1 & 0 \\ 0 & q\end{array}\right), q^{\frac{1}{4}}\right] \in \mathcal{G}$ and where $\left\{A_{j}^{*}\right\}$ is a complete set of coset representatives for $\left(\xi^{-1} \Gamma_{1}(4 N)^{*} \xi \cap \Gamma_{1}(4 N)^{*}\right) \backslash \Gamma_{1}(4 N)^{*}$. For a positive integer $m$ we define the shift operator $B_{m}$ by

$$
f\left|B_{m}=m^{-\frac{k}{4}} f\right|\left[\left(\begin{array}{cc}
m & 0 \\
0 & 1
\end{array}\right), m^{-\frac{1}{4}}\right] \text {. }
$$

The action of these two operators in terms of Fourier coefficients is easily described (see [10]): if $f \in S_{k / 2}(4 N, \psi)$ with $f(\tau)=$ 
$\sum_{n \geq 1} a(n) x^{n} \quad\left(x=e^{2 \pi i \tau}\right)$, then

$$
f \mid T_{q}(\tau)=\sum_{n \geq 1} a(n q) x^{n} \text { and } f \mid B_{m}(\tau)=\sum_{n \geq 1} a(n) x^{n m}
$$

Elementary properties of these operators can be found in Proposition 4.2 .

As in [10], for a positive integer $t$, we let $\chi_{t}$ denote the primitive character of order $\leq 2$ corresponding to the quadratic extension $\mathbb{Q}(\sqrt{t}) / \mathbb{Q}$.

\section{The Correspondence Between Newforms of Integral} and Half-Integral Weight. For an integer $\ell \geq 2$, a positive integer $M$ and a Dirichlet character $\psi$ defined modulo $M$, let $S_{\ell}^{-}(M, \psi)$ (resp. $\left.S_{\ell}^{+}(M, \psi)\right)$ denote the subspace of $S_{\ell}(M, \psi)$ generated by the oldforms (resp. newforms) (e.g. see [7]). With the notation as in $\S 1$, let $S_{k / 2}^{-}(4 N, \psi)$ (resp. $\left.S_{k / 2}^{+}(4 N, \psi)\right)$ denote the subspace of $S_{k / 2}(4 N, \psi)$ generated by the oldforms (resp. newforms) (e.g. see [11]). In addition, when $N$ is odd and squarefree and $\psi$ is an even quadratic character we have the Kohnen subspaces of oldforms and newforms which we shall denote by $S_{k / 2}^{-}(4 N, \psi)_{K}$ and $S_{k / 2}^{+}(4 N, \psi)_{K}$ respectively (see $[\mathbf{6}]$ ).

Let $F$ be a newform of integral weight with eigenvalues $\lambda_{p}$ for all the Hecke operators $T_{p}, p$ a prime. Denote by

$$
\begin{aligned}
& S_{k / 2}(4 N, \psi, F) \\
& \quad=\left\{f \in S_{k / 2}(4 N, \psi): f \mid T_{p^{2}}=\lambda_{p} f \text { for almost all primes } p\right\} .
\end{aligned}
$$

In Lemma 7 of [13], Shimura shows that

$$
S_{k / 2}(4 N, \psi)=\oplus S_{k / 2}(4 N, \psi, F)
$$

where the sum is over a finite number of newforms $F$ of weight $k-1$ and level dividing $2 N$, and that these summands are orthogonal with respect to the Petersson inner product. Actually, Shimura proves this for $k \geq 5$, but the result remains valid (see [16]) given our definition of $S_{3 / 2}(4 N, \psi)$ (see $\left.\S 1\right)$.

The spaces $S_{k / 2}(4 N, \psi, F)$ are of great interest. Flicker [2] (cf. [20]) gives conditions in terms of an automorphic representation of $F$ for the existence of an integer $N$ for which $S_{k / 2}(4 N, \psi, F) \neq\{0\}$, 
but gives no indication of the value of $N$. Vigneras [19] remarks that Flicker's conditions always hold when $F$ is a newform with trivial character. Waldspurger [20] gives a rather complex description of a basis for $S_{k / 2}(4 N, \psi, F)$ when it is nonzero, but he too does not determine for which values of $N, S_{k / 2}(4 N, \psi, F) \neq\{0\}$.

We begin this section by determining some conditions relating the level of $F$ to the value of $N$ under which $S_{k / 2}(4 N, \psi, F) \neq\{0\}$. In fact, we give conditions under which there are nonzero newforms in $S_{k / 2}(4 N, \psi, F)$. We first require some elementary properties of the Shimura lift.

2.1. Properties of the Shimura Lift. Let $f \in S_{k / 2}(4 N, \psi)$ with Fourier expansion $f(\tau)=\sum_{n \geq 1} a(n) x^{n}\left(x=e^{2 \pi i \tau}\right)$. Let $t$ be a squarefree positive integer and let $\psi_{t}^{(\lambda)}$ be the character defined modulo $4 N t$ given by $\psi_{t}^{(\lambda)}(m)=\psi(m)(-1 / m)^{\lambda}(t / m)$. Recall the $t$-Shimura lift of $f$ is defined by

$$
\operatorname{Sh}_{t}(f)=\sum_{n \geq 1} A_{t}(n) x^{n}
$$

where the Fourier coefficients $A_{t}(n)$ are determined by the product of two Dirichlet series:

$$
\sum_{n \geq 1} A_{t}(n) n^{-s}=L\left(s-\lambda+1, \psi_{t}^{(\lambda)}\right) \sum_{m \geq 1} a\left(t m^{2}\right) m^{-s} .
$$

For future reference we note that

$$
A_{t}(n)=\sum_{d \mid n} \psi_{t}^{(\lambda)}(d) d^{\lambda-1} a\left(t n^{2} / d^{2}\right)
$$

From [14], [9], [1], we know that the Shimura lift has the property that $\mathrm{Sh}_{t}(f) \in S_{k-1}\left(2 N, \psi^{2}\right)$.

LEMMA 2.1. Let $f=\sum_{n \geq 1} a(n) x^{n} \in S_{k / 2}(4 N, \psi)$, and let $t$ be a squarefree positive integer. If $\operatorname{Sh}_{t}(f)=\sum_{n \geq 1} A_{t}(n) x^{n}=0$, then $a\left(t n^{2}\right)=0$ for all $n \geq 1$.

Proof. $\mathrm{Sh}_{t}(f)=0$ if and only if $A_{t}(n)=0$ for all $n \geq 1$. From equation (1) above we have that $a(t)=A_{t}(1)=0$. Now suppose that $a\left(t n^{2}\right)=0$ for all positive integers $n$ such that $n$ is the product 
of fewer than $r$ primes (counting multiplicities). We have established this result with $r=1$. For $p$ a prime, equation (1) implies that

$$
0=A_{t}(n p)=a\left(t n^{2} p^{2}\right)+\sum_{\substack{d \mid n p \\ d>1}} \psi_{t}^{(\lambda)}(d) d^{\lambda-1} a\left(t n^{2} p^{2} / d^{2}\right)
$$

which completes the proof by induction.

Proposition 2.2. Let $f$ be a nonzero element of $S_{k / 2}(4 N, \psi)$. Then there exist an infinite number of squarefree positive integers $t$ such that $\mathrm{Sh}_{t}(f) \neq 0$.

Proof. If $\mathrm{Sh}_{t}(f)=0$ for all but a finite number of squarefree positive integers $t$, then by Lemma 2.1, the Fourier coefficients of $f$ are supported on only a finite number of square classes. By Theorem 3 of [18] the weight of $f$ must be $1 / 2$ of $3 / 2$, and at weight $3 / 2$, $f$ must be in the span of the theta series $h_{\psi}$, contrary to assumption.

\subsection{Odd squarefree levels and connections to the Kohnen} subspace. Since the definitions of the spaces of oldforms and newforms in the Kohnen subspace differ from those in the full space of half-integral weight cusp forms, it is apriori unclear what connections, if any, exist between newforms of these two types. We begin with a comparison of these spaces of newforms.

In Theorem 2 of [6], Kohnen defines a lifting which (for $N$ odd and squarefree and $\psi$ even quadratic) maps $S_{k / 2}^{+}(4 N, \psi)_{K}$ isomorphically onto $S_{k-1}^{+}(N, 1)$ and which commutes with the action of the respective Hecke operators. As an immediate consequence, we have

Proposition 2.3. Let $N$ be a squarefree odd positive integer, $\psi$ an even quadratic character defined modulo $4 N$. Then $S_{k / 2}^{+}(4 N, \psi)_{K}$ $\subset \oplus S_{k / 2}(4 N, \psi, F)$ where the sum is over all the normalized newforms $F \in S_{k-1}^{+}(N, 1)$.

For notational convenience, we set

$$
S_{k / 2}^{ \pm}(4 M, \psi, F)=S_{k / 2}(4 M, \psi, F) \cap S_{k / 2}^{ \pm}(4 M, \psi) .
$$

THEOREM 2.4. Suppose that $N$ is an odd positive integer and $\psi$ is an even Dirichlet character defined modulo $4 N$. Let $F \in$ 
$S_{k-1}^{+}\left(N, \psi^{2}\right) \cup S_{k-1}^{+}\left(2 N, \psi^{2}\right)$ be a normalized newform, and suppose that $S_{k / 2}(4 D, \psi, F) \neq\{0\}$ for some $D \mid N$. Then

(1) $D=N$;

(2) $S_{k / 2}^{-}(4 N, \psi, F)=\{0\}$, and so $S_{k / 2}(4 N, \psi, F) \subset S_{k / 2}^{+}(4 N, \psi)$;

(3) If $N$ is squarefree and $\psi^{2}=1$, then $S_{k / 2}^{+}(4 N, \psi)_{K} \subset S_{k / 2}^{+}(4 N, \psi)$.

REMARKS. Loosely put, part (1) of the theorem says that $S_{k / 2}(4 D, \psi, F)=\{0\}$ if $D$ is small relative to the level of the newform $F$. Alternatively, it says that if a half-integral weight cusp form lifts to a newform, then its level can't be too small relative to the level of the newform. Moreover, the result holds for any even "square root" of $\psi^{2}$, i.e., with $\psi$ replaced by $\psi \chi$ for any even quadratic $\chi$. Part (2) says that any simultaneous eigenform of level $4 N$ which has almost all the same Hecke eigenvalues as a newform of level $N$ or $2 N$ must be a newform itself. Part (3) concerns the fact that newforms in the Kohnen subspace are defined differently (since oldforms are) than newforms for the full space of cusp forms. The results says that despite this difference, the spaces of newforms are still related.

Proof. (1) Let $f \in S_{k / 2}(4 D, \psi) \cap S_{k / 2}(4 N, \psi, F)=S_{k / 2}(4 D, \psi, F)$, $f \neq 0$. We may assume that $4 D$ is the exact level of $f$. By Proposition 2.2, we can choose a positive squarefree integer $t$ such that $\mathrm{Sh}_{t}(f) \neq 0$. From [14], [9], and [1] we know that $\mathrm{Sh}_{t}(f) \in$ $S_{k / 2}\left(2 D, \psi^{2}\right)$, and since the Shimura lift commutes with the action of the Hecke operators for primes not dividing the level, we have that $\mathrm{Sh}_{t}(f)$ and $F$ have the same eigenvalues for all Hecke operators $T_{p}$ for $p$ a prime $p \nmid 2 N$. Since $F$ is a newform, the level of $F$ divides that of $\operatorname{Sh}_{t}(f)$. Thus $N \mid 2 D$ or $2 N \mid 2 D$ which, since $N$ is odd, implies in either case that $N \mid D$, and hence the result.

(2) Let $f \in S_{k / 2}^{-}(4 N, \psi, F)$. Then $f=\sum g_{i}$ where the $g_{i}$ are oldforms, that is each $g_{i}$ is a simultaneous eigenform for almost all of the Hecke operators $T_{p^{2}}$ with $g_{i}=h_{i} \mid B_{\ell_{i}}$ for some $h_{i} \in S_{k / 2}\left(4 N_{i}, \psi \chi_{\ell_{i}}\right)$ where $N_{i}<N$ and $\ell_{i} N_{i} \mid N$ (see [11]). Now each $g_{i}$ is an element of $S_{k / 2}(4 N, \psi, H)$ for some integral weight newform $H$, and $S_{k / 2}(4 N, \psi)=\oplus S_{k / 2}(4 N, \psi, H)$, the (orthogonal) sum over newforms $H$ ([13, Lemma 7]). Thus given that $f \in S_{k / 2}(4 N, \psi, F)$, we may assume that each $g_{i} \in S_{k / 2}(4 N, \psi, F)$. It follows from Lemma 3 of $[\mathbf{1 0}]$, that the $h_{i}$ are also simultaneous eigenforms for almost 
all of the Hecke operators $T_{p^{2}}$ having the same eignevalues as the $g_{i}$. Thus each $h_{i} \in S_{k / 2}\left(4 N_{i}, \psi \chi_{\ell_{i}}, F\right)$. Since $N_{i}<N$ we have that $h_{i}=0$ by part (1) of this theorem. Thus $S_{k / 2}^{-}(4 N, \psi, F)=\{0\}$ and hence $S_{k / 2}(4 N, \psi, F) \subset S_{k / 2}^{+}(4 N, \psi)$.

(3) From Proposition 2.3 and part (2) of this theorem, we have that $S_{k / 2}^{+}(4 N, \psi)_{K} \subset \oplus S_{k / 2}(4 N, \psi, F) \subset S_{k / 2}^{+}(4 N, \psi)$.

Corollary 2.5. Let $N$ be an odd squarefree positive integer, and $F \in S_{k-1}^{+}(N, 1)$ a newform. Then for any even quadratic character $\psi$ defined modulo $4 N$, we have:

(1) $S_{k / 2}(4 D, \psi, F)=\{0\}$ for all $D \mid N, D<N$;

(2) $S_{k / 2}^{+}(4 N, \psi, F) \neq\{0\}$;

(3) $S_{k / 2}(4 N M, \psi, F) \neq\{0\}$ for all $M \geq 1$.

Proof. The first item is a restatement of part (1) of Theorem 2.4. For (2), we observe that by Theorem 2 of $[\mathbf{6}], S_{k-1}^{+}(N, 1) \cong$ $S_{k / 2}^{+}(4 N, \psi)_{K} \subset S_{k / 2}(4 N, \psi)$, where the isomorphism is as modules for the respective Hecke algebras. It follows that $S_{k / 2}(4 N, \psi, F) \neq$ $\{0\}$, and hence by part (3) of Theorem 2.4, $S_{k / 2}^{+}(4 N, \psi)_{K}$ $\subset S_{k / 2}^{+}(4 N, \psi)$, whence $S_{k / 2}^{+}(4 N, \psi, F) \neq\{0\}$. The third part of the corollary is immediate since $S_{k / 2}(4 N, \psi, F) \subset S_{k / 2}(4 N M, \psi, F)$ for all $M \geq 1$.

REMARK. The more interesting question suggested by the corollary is whether the subspace of newforms, $S_{k / 2}^{+}(4 N M, \psi, F)$, is nonzero for any $M>1$. Rather suprisingly, the answer is yes, and for arbitrarily large $M$, as we shall see in $\S 2.5$.

We conclude this section with a simple result concerning eigenvalues of newforms corresponding to Hecke operators $T_{q^{2}}$ for primes $q$ dividing the level $N$. We recall that since we cannot characterize newforms up to scalar multiples by their Hecke eigenvalues, it is not clear that a newform is an eigenform for any of the Hecke operators $T_{q^{2}}$ for primes $q$ dividing the level $N$. A result in this direction (cf. Theorem 3 of $\operatorname{Li}[7])$ is

Corollary 2.6. Let $f$ be a newform in $S_{k / 2}(4 N, \psi)$ and suppose that $q$ is a prime such that $q^{2} \mid N$ and $\psi \chi_{q}$ is defined modulo $4 N / q$. (1) If $f$ is an eigenform for $T_{q^{2}}$, then $f \mid T_{q^{2}}=0$. 
(2) If $N$ is odd, and $f \in S_{k / 2}(4 N, \psi, F)$ for a newform $F$ of weight $k-1$ and (exact) level $N$ or $2 N$, then $f$ is an eigenform for $T_{q^{2}}$ and $f \mid T_{q^{2}}=0$.

REMARK. Regarding the hypotheses of (2), we know that $f$ corresponds to a newform of level dividing $2 N$, but not that the exact level is divisible by $N$.

Proof. In either case $f \mid T_{q^{2}} \in S_{k / 2}(4 N / q, \psi)$ (see Proposition 3.2 below). If $f$ is an eigenform for the Hecke operator $T_{q^{2}}$, then $f \mid T_{q^{2}}=$ $\lambda_{q} f$. If $\lambda_{q} \neq 0$, we have a contradiction to the exact level of $f$ being $4 N$. In the second case, we see that $f \mid T_{q^{2}} \in S_{k / 2}(4 N / q, \psi, F)$, since $T_{q^{2}}$ and $T_{p^{2}}$ commute for primes $p \neq q([\mathbf{1 0}$, Lemma 1]). By part (1) of Theorem 2.4, $S_{k / 2}(4 N / q, \psi, F)=\{0\}$, hence the result.

2.3. More general levels. In this section let $N=2^{\mu} N_{0}$ where $N_{0}$ is odd and squarefree, and $0 \leq \mu \leq 2$. Let $\psi$ be any even quadratic Dirichlet character defined modulo $4 N$, with the conductor of $\psi$ divisible by 8 if $\mu=2$. The corollary in $\S 3$ of [16] may be stated as

THEOREM 2.7. (Ueda).

(1) $S_{k / 2}(4 N, \psi) \cong S_{k-1}(2 N, 1)$;

(2) $S_{k / 2}(4 N, \psi)_{K} \cong S_{k-1}(N, 1)$ if $\mu=0$

where the isomorphisms are as modules for the respective Hecke algebras.

We use this to show

THEOREM 2.8. With $N$ and $\psi$ as above, let $F$ be a newform in $S_{k-1}(2 N, 1)$. Then

(1) $S_{k / 2}(4 D, \psi, F)=\{0\}$ for all $D \mid N, D<N$;

(2) $S_{k / 2}^{+}(4 N, \psi, F) \neq\{0\}$;

(3) $S_{k / 2}(4 N M, \psi, F) \neq\{0\}$ for all $M \geq 1$.

Proof. First observe that by the definition of the space of oldforms (see [11]) and by Theorem 2.7 above, we have

$$
\begin{aligned}
S_{k / 2}^{-}(4 N, \psi)= & \sum_{\substack{q|N \\
\operatorname{cond}(\psi)| 4 N / q}} S_{k / 2}(4 N / q, \psi) \\
& +\sum_{\substack{q|N \\
\operatorname{cond}(\psi \chi q)| 4 N / q}} S_{k / 2}\left(4 N / q, \psi \chi_{q}\right) \mid B_{q}
\end{aligned}
$$




$$
\begin{aligned}
& \cong \sum_{\substack{q|N \\
\operatorname{cond}(\psi)| 4 N / q}} S_{k-1}(2 N / q, 1) \\
& +\sum_{\substack{q|N \\
\operatorname{cond}(\psi \times q)| 4 N / q}} S_{k-1}(2 N / q, 1)
\end{aligned}
$$

where the sums are over primes $q$ dividing $N$ and where we observe that the shift operator $B_{q}$ induces an isomorphism as modules for the Hecke algebra (see Lemma $3 \mathrm{~d}$ of [10]) between $S_{k / 2}\left(4 N / q, \psi \chi_{q}\right)$ and $S_{k / 2}\left(4 N / q, \psi \chi_{q}\right) \mid B_{q}$. Thus any form in $S_{k / 2}^{-}(4 N, \psi, F)$ is equivalent to (has the same Hecke eigenvalues as) a cusp form of level $2 N / q$ and trivial character for some prime $q \mid N$. On the other hand, such a cusp form is equivalent to the newform $F$ of level $2 N$, hence $S_{k / 2}^{-}(4 N, \psi, F)=\{0\}$. Since for any $D \mid N, D<N$, we have $S_{k / 2}(4 D, \psi) \subset S_{k / 2}^{-}(4 N, \psi)$, the first assertion follows.

Since $F \in S_{k-1}(2 N, 1)$, Theorem 2.7 implies that $S_{k / 2}(4 N, \psi, F) \neq$ $\{0\}$, and since $S_{k / 2}^{-}(4 N, \psi, F)=\{0\}$, we must have that $S_{k / 2}^{+}(4 N, \psi, F) \neq\{0\}$, hence (2). The third part is clear.

2.4. Explicit decomposition of newform spaces. Newforms of half-integral weight cannot be characterized by their Hecke eigenvalues $([\mathbf{5}])$, which means that a multiplicity-one theorem does not hold for the space of half-integral weight newforms. In the results below, we give more precise information about the multiplicities with which newforms occur, by giving an isomorphism which explicitly decomposes a given space of half-integral weight newforms into a direct sum of spaces of integral weight newforms. This not only demonstrates explicitly that the multiplicities are either one or two, but also suggests that the mutliplicity two occurrences are related to the Kohnen subspaces.

We first consider two special cases.

Proposition 2.9. Let $p$ be an odd prime and $\psi$ an even quadratic Dirichlet character defined modulo $4 p$. Then

$$
S_{k / 2}^{+}(4 p, \psi) \cong S_{k-1}^{+}(2 p, 1) \oplus 2 S_{k-1}^{+}(p, 1) \oplus S_{k-1}^{+}(2,1) \oplus 2 S_{k-1}^{+}(1,1)
$$


Proof. It is easy to check that the only possibilities for $\psi$ are $\psi=1$ or $\chi_{p}$, and that

$$
\begin{aligned}
S_{k / 2}^{-}(4 p, \psi) & = \begin{cases}S_{k / 2}(4,1), & \text { if } \psi=1 \\
S_{k / 2}(4,1) \mid B_{p}, & \text { if } \left.\psi \neq 1 \text { (i.e. } \psi=\chi_{p}\right)\end{cases} \\
& \cong S_{k / 2}(4,1) \\
& \cong S_{k-1}(2,1) \\
& \cong S_{k-1}^{+}(2,1) \oplus 2 S_{k-1}^{+}(1,1)
\end{aligned}
$$

where the first isomorphism is given by $B_{p}$, the second isomorphism is given by Theorem 2.7, and the third by the theory of integral weight newforms (see $\mathrm{Li}[7]$ ).

Also from Theorem 2.7 and newform theory for integral weight forms, we have that

$$
\begin{aligned}
S_{k / 2}(4 p, \psi) & \cong S_{k-1}(2 p, 1) \\
& \cong S_{k-1}^{+}(2 p, 1) \oplus 2 S_{k-1}^{+}(p, 1) \oplus 2 S_{k-1}^{+}(2,1) \oplus 4 S_{k-1}^{+}(1,1) .
\end{aligned}
$$

Moreover, by definition and the characterization of the oldform space above, we have that

$$
\begin{aligned}
S_{k / 2}(4 p, \psi) & =S_{k / 2}^{+}(4 p, \psi) \oplus S_{k / 2}^{-}(4 p, \psi) \\
& \cong S_{k / 2}^{+}(4 p, \psi) \oplus S_{k-1}^{+}(2,1) \oplus 2 S_{k-1}^{+}(1,1) .
\end{aligned}
$$

Comparing the two expressions for $S_{k / 2}(4 p, \psi)$ and cancelling like terms (semisimplicity of the Hecke algebras) yields the result.

REMARKS. (1) Note that by Theorem 2.4, $S_{k / 2}^{+}(4 p, \psi)_{K} \subset$ $S_{k / 2}^{+}(4 p, \psi)$, and from Kohnen's Theorem $2[6]$ that $S_{k / 2}^{+}(4 p, \psi)_{K}$ $\cong S_{k-1}^{+}(p, 1)$, thus the Kohnen subspace contributes one of the two copies of $S_{k-1}^{+}(p, 1)$ in $S_{k / 2}^{+}(4 p, \psi)$.

(2) If $k<13$ or $k=15$ then $S_{k-1}(1,1)=\{0\}$, so that the nonKohnen newforms all occur with multiplicity one.

We next consider a slightly more general level, but restrict to trivial character. For notational ease, we write $S_{\ell}(M)$ for $S_{\ell}(M, 1)$.

Proposition 2.10. Let $p$ and $q$ be distinct odd primes. Then

$$
\begin{array}{r}
S_{k / 2}^{+}(4 p q) \cong S_{k-1}^{+}(2 p q) \oplus S_{k-1}^{+}(2 p) \oplus S_{k-1}^{+}(2 q) \oplus 2 S_{k-1}^{+}(p q) \\
\oplus 2 S_{k-1}^{+}(p) \oplus 2 S_{k-1}^{+}(q) \oplus S_{k-1}^{+}(2) \oplus 2 S_{k-1}^{+}(1) .
\end{array}
$$


Remark. As in Proposition 2.9, we note that one of the factors, $S_{k-1}^{+}(p q)$, contributing to a multiplicity two occurrence in the decomposition of $S_{k / 2}^{+}(4 p q)$ is expected as it corresponds to the subspace of Kohnen newforms of level $4 \mathrm{pq}$ (i.e. to $S_{k / 2}^{+}(4 p q)_{K} \subset$ $\left.S_{k / 2}^{+}(4 p q)\right)$. In Theorem 2.12, we offer a possible explanation for the other factors which occur with multiplicity 2 .

Before proceeding with the proof of the proposition, it is convenient to first characterize the space of oldforms in

LEMMA 2.11. With the notation as above,

$$
S_{k / 2}^{-}(4 p q)=S_{k / 2}^{+}(4 p) \oplus S_{k / 2}^{+}(4 q) \oplus S_{k / 2}^{+}(4) .
$$

Proof. By definition,

$$
\begin{aligned}
S_{k / 2}^{-}(4 p q) & =S_{k / 2}(4 p)+S_{k / 2}(4 q) \\
& =S_{k / 2}^{+}(4 p)+S_{k / 2}^{-}(4 p)+S_{k / 2}^{+}(4 q)+S_{k / 2}^{-}(4 q) \\
& =S_{k / 2}^{+}(4 p)+S_{k / 2}^{+}(4 q)+S_{k / 2}^{+}(4)
\end{aligned}
$$

where the last equality follows from the characterization of $S_{k / 2}^{-}(4 \ell)$ in the proof Proposition 2.9 ( $\ell$ an odd prime). Since $S_{k / 2}^{+}(4)=$ $S_{k / 2}(4) \subset S_{k / 2}^{-}(4 p) \cap S_{k / 2}^{-}(4 q)$, it is orthogonal to the corresponding spaces of newforms, hence in particular,

$$
S_{k / 2}^{-}(4 p q)=\left[S_{k / 2}^{+}(4 p)+S_{k / 2}^{+}(4 q)\right] \oplus S_{k / 2}^{+}(4) .
$$

To see that the bracketed sum is direct, we first note that in a manner exactly as in the integral weight case (see e.g. Lemma 7 of $[7])$, we have that

$$
S_{k / 2}(4 N, \psi) \cap S_{k / 2}(4 M, \psi) \subset S_{k / 2}(4 D, \psi)
$$

where $D=\operatorname{gcd}(M, N)$. Thus if $f \in S_{k / 2}^{+}(4 p) \cap S_{k / 2}^{+}(4 q)$, we must have $f \in S_{k / 2}(4)=S_{k / 2}^{-}(4 p)=S_{k / 2}^{-}(4 q)$, hence $f=0$, and the lemma is established.

Proof of the Proposition. By Theorem 2.7 and the theory of newforms, we have that

$$
\begin{aligned}
S_{k / 2}(4 p q) \cong & S_{k-1}(2 p q) \\
\cong & S_{k-1}^{+}(2 p q) \oplus 2\left(S_{k-1}^{+}(2 p) \oplus S_{k-1}^{+}(2 q) \oplus S_{k-1}^{+}(p q)\right) \\
& \oplus 4\left(S_{k-1}^{+}(p) \oplus S_{k-1}^{+}(q) \oplus S_{k-1}^{+}(2)\right) \oplus 8 S_{k-1}^{+}(1) .
\end{aligned}
$$


On the other hand, by definition and Lemma 2.11, we have

$$
\begin{aligned}
S_{k / 2}(4 p q) & =S_{k / 2}^{+}(4 p q) \oplus S_{k / 2}^{-}(4 p q) \\
& =S_{k / 2}^{+}(4 p q) \oplus S_{k / 2}^{+}(4 p) \oplus S_{k / 2}^{+}(4 q) \oplus S_{k / 2}^{+}(4)
\end{aligned}
$$

From Theorem 2.7 and newform theory, we have $S_{k / 2}^{+}(4)=S_{k / 2}(4)$ $\cong S_{k-1}(2) \cong S_{k-1}^{+}(2) \oplus 2 S_{k-1}^{+}(1)$. Finally, applying Proposition 2.9 twice in the above expression yields

$$
\begin{aligned}
S_{k / 2}(4 p q) \cong & S_{k / 2}^{+}(4 p q) \oplus S_{k-1}^{+}(2 p) \oplus 2 S_{k-1}^{+}(p) \oplus S_{k-1}^{+}(2) \oplus 2 S_{k-1}^{+}(1) \\
& \oplus S_{k-1}^{+}(2 q) \oplus 2 S_{k-1}^{+}(q) \oplus S_{k-1}^{+}(2) \oplus 2 S_{k-1}^{+}(1) \\
& \oplus S_{k-1}^{+}(2) \oplus 2 S_{k-1}^{+}(1)
\end{aligned}
$$

Comparing the two expressions for $S_{k / 2}(4 p q)$ and cancelling like terms (semisimplicity of the Hecke algebras) yields the result.

For the case of trivial character, we summarize these two propositions and offer an interpretation of the extra terms which contribute the extra multiplicity to the newform decomposition.

THEOREM 2.12. Let $N$ be an odd prime or the product of two distinct odd primes. Then

$$
\begin{aligned}
S_{k / 2}^{+}(4 N) & \cong \oplus_{d \mid 2 N} S_{k-1}^{+}(d) \oplus \oplus_{e \mid N} S_{k-1}^{+}(e) \\
& \cong \oplus_{d \mid N}\left[S_{k-1}^{+}(2 d) \oplus 2 S_{k-1}^{+}(d)\right] \\
& \cong \oplus_{d \mid 2 N} S_{k-1}^{+}(d) \oplus \oplus_{e \mid N} S_{k / 2}^{+}(4 e)_{K}
\end{aligned}
$$

Proof. The first statement is just a reformulation of Propositions 2.9 and 2.10. The third statement is immediate from Theorem 2 of [6] since $S_{k / 2}^{+}(4 e, 1)_{K} \cong S_{k-1}^{+}(e, 1)$.

REMARKS. (1) The first two decompositions make clear that newforms occur with multiplicity one or two and clearly identifies which newforms occur with a given multiplicity.

(2) The third decomposition is meant to be suggestive. It seems far too remarkable to be coincidence that the spaces contributing to the multiplicity two part of the decomposition of the space of 
newforms $S_{k / 2}^{+}(4 N)$ should correspond precisely to the direct sum of all possible Kohnen subpaces of newforms of levels dividing $4 \mathrm{~N}$, but we can offer no explanation for this phenomenon at present. Moreover, we note that excluding the "Kohnen" contribution, the remaining forms satisfy a multiplicity-one theorem. It also seems appropriate to make the following conjecture:

ConjeCture 2.13. Theorem 2.12 is true for $N$ any odd squarefree positive integer. ${ }^{\dagger}$

REMARKS. The conjecture will follow from the lemma that $S_{k / 2}^{-}(4 N)=\oplus_{\substack{d \mid N \\ d<N}} S_{k / 2}^{+}(4 d)$. It follows easily from the definition and induction on the number of prime divisors of $N$ that $S_{k / 2}^{-}(4 N)=$ $\sum_{\substack{d \mid N \\ d<N}} S_{k / 2}^{+}(4 d)$. From here, it suffices to show that $\sum_{d} S_{k / 2}^{+}(4 d)=$ $\oplus_{d} S_{k / 2}^{+}(4 d)$ where the sums are over divisors $d \mid N$ having a fixed number of prime divisors.

2.5. Conditions when $S_{k / 2}^{+}(4 N, \psi, F) \neq\{0\}$. In Corollary 2.5 and Theorem 2.8, we have given conditions which force $S_{k / 2}^{+}(4 N, \psi, F)=\{0\}$ when the level of $F$ is large relative to $N$. The far more interesting situation is when the level of $F$ is small relative to $N$.

As an immediate consequence of Propositions 2.9 and 2.10, we are able to give conditions under which $S_{k / 2}^{+}(4 N, \psi, F) \neq 0$ for values of $N$ which are arbitrarily large. In particular, this says that the levels $N$ for which $S_{k / 2}^{+}(4 N, \psi, F) \neq\{0\}$ are not determined by the level of the newform $F$. Thus in general, the level of a half-integral weight newform is not characterized either by the level of the newform to which it corresponds, or by the eigenvalues of that newform. This of course is in striking contrast to those newforms in the Kohnen subspace.

\section{THEOREM 2.14 .}

(1) If $F \in S_{k-1}^{+}(2) \cup S_{k-1}^{+}(1)$ is a newform, then $S_{k / 2}^{+}(4 p, \psi, F) \neq$ $\{0\}$ for all odd primes $p$ and for any even quadratic Dirichlet character $\psi$ defined modulo $4 p$.

${ }^{\dagger}$ Added in proof: This conjecture has recently been established by the first author, and its proof will appear elsewhere. 
(2) If $p$ is an odd prime and $F$ is a newform of weight $k-1$ and any level dividing $2 p$, then for any odd prime $q \neq p$, $S_{k / 2}^{+}(4 p q, 1, F) \neq\{0\}$.

Proof. The decompositions given in Propositions 2.9 and 2.10 say that to each nonzero integral weight newform appearing on the right hand side of the decomposition, there exists a nonzero half-integral weight newform appearing on the left hand side of the decomposition which has the same Hecke eigenvalues at almost all primes. This establishes the result.

This result is rather surprising since the newforms which arise in this way do not seem to be produced by any "natural" operations on cusp forms. For example, an obvious way in which to attempt to produce a newform of arbitrarily high level, with almost all the same Hecke eigenvalues as a given newform, is by means of character twists. While character twists do not explain the results of the theorem above, we briefly discuss their properties to justify this assertion and to indicate their significance.

Recall that if $f(\tau)=\sum a(n) x^{n} \in S_{k / 2}(4 N, \psi)$ and if $\chi$ is a Dirichlet character with conductor $r$, then the twist of $f$ by $\chi$ is the cusp form $f_{\chi}(\tau)=\sum \chi(n) a(n) x^{n} \in S_{k / 2}\left(4 N r^{2}, \psi \chi^{2}\right)$ (see $\S 6$ of [11]). Moreover, if $f \in S_{k / 2}(4 N, \psi)$ is an eigenform for the Hecke operator $T_{p^{2}}(p \nmid 4 N)$ with eigenvalue $\lambda_{p}$, then $f_{\chi}$ is an eigenform with eigenvalue $\chi(p)^{2} \lambda_{p}$. Thus if the goal is to generate newforms of arbitrarily high level having the same eigenvalues as a given newform, the character $\chi$ must be quadratic.

If we assume that $\operatorname{gcd}(r, 4 N)=1$, and $f \in S_{k / 2}^{+}(4 N, \psi)$ is a newform, then by Theorem 6.6 of [11], the exact level of $f_{\chi}$ is $4 N r^{2}$. Hence even if $f_{\chi}$ is a newform, its level is no longer squarefree. The examples we produced above all have the feature that the level is of the form $4 N M$ where $M$ is odd and squarefree, thus character twists do not explain the existence of these newforms.

3. Multiplicity-One Theorems. In the case of integral weight modular forms, we know that it is possible to have two linearly independent cusp forms which have the same eigenvalues for all but a finite number of the Hecke operators $T_{p}, p$ a prime. However, if we restrict to the subspace of newforms, then this collection of eigenvalues is sufficient to characterize a cusp form up to a constant 
multiple. Moreover, as a consequence of the multiplicity-one theorem for newforms of integral weight, any newform which a priori is a simultaneous eigenform (only) for all but a finite number of the Hecke operators, is in fact a simultaneous eigenform for all of the Hecke operators.

In the half-integral weight case, a knowledge of the Hecke eigen. values, even for all of the Hecke operators $T_{p^{2}}$ is far from sufficient, to characterize a cusp form. Such information is at first glance ad equate only to determine the values of the Fourier coefficients of a cusp form $f$ which lie in a given square class. That is, given the value of the Fourier coefficient $a(t)$ for $t$ a squarefree positive integer, the values of $a\left(t n^{2}\right)$ are determined by the Hecke eigenvalues. What is lacking is a knowledge of the values of the Fourier coefficients $a(t)$ for squarefree integers $t$. Although Waldspurger [20] is able (under mild assumptions) to characterize the value of $a(t)^{2}$, the sign of the coefficient is undetermined. Moreover, by Theorem 3 of [18], we know that $f$ must be supported on an infinite number of square classes, so that determining the actual values of the $a\left(t n^{2}\right)$ for a given cusp form always involves an infinite number of choices of sign. The need to deal with this ambiguity motivates the results below in which we develop sufficient conditions to characterize half-integral weight newforms.

It is obvious that a knowledge of all of the Hecke eigenvalues and of all the values $a(t)$ for squarefree $t$ is sufficient to characterize all of the Fourier coefficients. In light of the comments above we first consider a special case in which it is assumed that we are working with simultaneous eigenforms for all of the Hecke operators $T_{p^{2}}$ and have a knowledge of almost all of the Fourier coefficients at squarefree values $t$. We find that this is more than enough information to characterize a cusp form.

Proposition 3.1 Let $N$ be a positive integer, and $\psi$ a Dirichlet. character defined modulo $4 N$. Suppose that $f, g \in S_{k / 2}(4 N, \psi)$ with Fourier expansions $f=\sum_{n \geq 1} a(n) x^{n}$ and $g=\sum_{n \geq 1} b(n) x^{n}$. Suppose that for all primes $p, f$ and $g$ are simultaneous eigenform.s for all of the Hecke operators $T_{p^{2}}$ having the same eigenvalues for all p. Moreover, suppose that the Fourier coefficients $a(t)=b(t)$ for 
almost all squarefree positive integers $t$. Then $f=g$.

Proof. Consider $h=f-g=\sum_{n \geq 1} c(n) x^{n}$. Since $h$ is a simultaneous eigenform for all of the Hecke operators $T_{p^{2}}$, it follows from the action of the Hecke operators on the Fourier coefficients that if $c(t)=0$, then $c\left(t n^{2}\right)=0$ for all positive integers $n$. If $h \neq 0$, then $c\left(t n^{2}\right)=0$ except for a (non-empty) finite set of positive squarefree integers $t$. By Theorem 3 of [18], $h$ has weight $1 / 2$ or $3 / 2$ and at weight $3 / 2$ lies in the span of the theta series $h_{\psi}$, contrary to assumption.

Notice that in the previous proposition, we have not assumed that the cusp forms are newforms. In the integral weight case, a newform is automatically a simultaneous eigenform for all of the Hecke operators, a consequence of multiplicity-one. For half-integral weight forms this is far from clear, for if $f \in S_{k / 2}(4 N, \psi, F)$ for some integral weight newform $F$ and $q$ is a prime dividing $N$, we know merely that $f \mid T_{q^{2}} \in S_{k / 2}(4 N, \psi, F)$ which would imply that $f$ is an eigenform for $T_{q^{2}}$ if $S_{k / 2}(4 N, \psi, F)$ is 1-dimensional, but not necessarily otherwise. In what follows, we add the constraint that our cusp form is a newform in exchange for the assumption that it is a simultaneous eigenform for all the Hecke operators. As we shall see, we again get a mutltiplicity-one theorem (see Theorem 3.9), however the proof requires significantly more work.

We first give some of the elementary properties of the Hecke and shift operators in

Proposition 3.2. Suppose $m \in \mathbb{Z}_{+}$and $q$ is a prime dividing $N$. Then $B_{m}$ maps $S_{k / 2}(4 N, \psi)$ to $S_{k / 2}\left(4 N m, \psi \chi_{m}\right)$ and $T_{q}$ maps $S_{k / 2}(4 N, \psi)$ to $S_{k / 2}\left(4 N, \psi \chi_{q}\right)$. Moreover, if $q^{2} \mid N$ and $\psi \chi_{q}$ is defined modulo $4 N / q$, then $T_{q}$ takes $S_{k / 2}(4 N, \psi)$ to $S_{k / 2}\left(4 N / q, \psi \chi_{q}\right)$ and hence $T_{q^{2}}=T_{q}^{2}$ takes $S_{k / 2}(4 N, \psi)$ to $S_{k / 2}(4 N / q, \psi)$.

Proof. Proofs of the first two statements can be found in [14] and [10]. The remaining statements are proven in analogy with Lemma 1 of $[7]$.

We now define an analogue of the Atkin-Lehner involution operator $W_{q}$. Versions of this operator have already been defined in [6] and in [8]; our definition differs from theirs by (at most) a constant. 
Definition. Let $q$ be a prime dividing $N$, and set $\alpha=\operatorname{ord}_{q} 4 N$. For $f \in S_{k / 2}(4 N, \psi)$, define $W_{q}=W_{q}^{4 N}$ by

$$
f\left|W_{q}=\left(\psi \chi_{q}^{\alpha}\right)_{q}(b) f\right|\left[\left(\begin{array}{cc}
1 & 0 \\
0 & q^{\alpha}
\end{array}\right), q^{\alpha / 4}\right] C^{*}
$$

where $C=\left(\begin{array}{cc}q^{\alpha} a & b \\ 4 N c / q^{\alpha} & 1\end{array}\right) \in \Gamma_{1}\left(4 N / q^{\alpha}\right)$, and $\left(\psi \chi_{q}^{\alpha}\right)_{q}$ is the $q$-part of the character $\psi \chi_{q}^{\alpha}$.

First we remark that even when $q=2, \theta(C \tau) / \theta(\tau)$ is an automorphy factor for $C$ since, following the proof of the transformation formula for theta functions (see [21]) we find that $\theta(C \tau) / \theta(\tau)=$ $(c+1 / \tau)^{1 / 2} \tau^{1 / 2}$. To show $W_{q}$ is well-defined, notice that if

$$
C=\left(\begin{array}{cc}
q^{\alpha} a & b \\
4 N c / q^{\alpha} & 1
\end{array}\right) \text { and } C^{\prime}=\left(\begin{array}{cc}
q^{\alpha} a^{\prime} & b^{\prime} \\
4 N c^{\prime} / q^{\alpha} & 1
\end{array}\right)
$$

are as in the definition of $W_{q}$, then

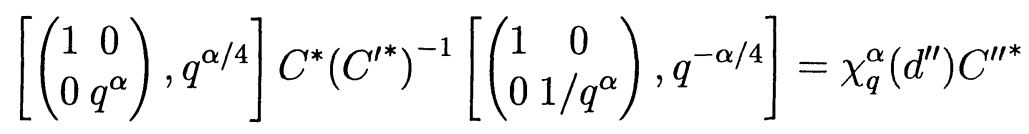

where

$$
C^{\prime \prime}=\left(\begin{array}{cc}
1 & 0 \\
0 & q^{\alpha}
\end{array}\right) C\left(C^{\prime}\right)^{-1}\left(\begin{array}{cc}
1 & 0 \\
0 & 1 / q^{\alpha}
\end{array}\right)=\left(\begin{array}{ll}
a^{\prime \prime} & b^{\prime \prime} \\
c^{\prime \prime} & d^{\prime \prime}
\end{array}\right) \in \Gamma_{0}(4 N) .
$$

Thus $W_{q}$ is well-defined if and only if

$$
\left(\psi \chi_{q}^{\alpha}\right)_{q}(b) f\left|C^{*}=\left(\psi \chi_{q}^{\alpha}\right)_{q}\left(b^{\prime}\right) f\right| C^{\prime *}
$$

or equivalently

$$
\left(\psi \chi_{q}^{\alpha}\right)_{q}\left(b / b^{\prime}\right) f \mid \chi_{q}^{\alpha}\left(d^{\prime \prime}\right) C^{\prime \prime *}=f .
$$

But $d^{\prime \prime} \equiv 1\left(\bmod 4 N / q^{\alpha}\right)$ and $d^{\prime \prime} \equiv-4 N c b^{\prime} / q^{\alpha}\left(\bmod q^{\alpha}\right)$, hence

$$
\left(\psi \chi_{q}^{\alpha}\right)_{q}\left(b / b^{\prime}\right) \psi \chi_{q}^{\alpha}\left(d^{\prime \prime}\right)=\left(\psi \chi_{q}^{\alpha}\right)_{q}\left(-4 N c b / q^{\alpha}\right)=1
$$

since $-4 N c b / q^{\alpha} \equiv 1 \quad\left(\bmod q^{\alpha}\right)$.

We now establish some basic properties of $W_{q}$. Consider the space $S_{k / 2}(4 N, \psi)$, and let $q$ be a prime dividing $N$. Set $\alpha=\operatorname{ord}_{q} 4 N$, 
$Q=q^{\alpha}$, and $M=4 N / Q$. Write $\psi=\psi_{Q} \psi_{M}$. With this notation, we have the

Proposition 3.3. The operator $W_{q}$ takes $S_{k / 2}\left(4 N, \psi_{Q} \psi_{M}\right)$ to $S_{k / 2}\left(4 N, \overline{\psi_{Q}} \psi_{M} \chi_{q}^{\alpha}\right)$.

Proof. Take $A^{\prime}=\left(\begin{array}{ll}a^{\prime} & b^{\prime} \\ c^{\prime} & d^{\prime}\end{array}\right) \in \Gamma_{0}(4 N)$, and $C=\left(\begin{array}{cc}q^{\alpha} a & b \\ 4 N c / q^{\alpha} & 1\end{array}\right) \in$ $\Gamma_{1}\left(4 N / q^{\alpha}\right)$ as in the definition of $W_{q}$. Then

$$
\left[\left(\begin{array}{cc}
1 & 0 \\
0 & q^{\alpha}
\end{array}\right), q^{\alpha / 4}\right] C^{*} A^{\prime} C^{-1^{*}}\left[\left(\begin{array}{cc}
1 & 0 \\
0 & 1 / q^{\alpha}
\end{array}\right), q^{-\alpha / 4}\right]=\chi_{q}^{\alpha}\left(d^{\prime \prime}\right) A^{\prime \prime *}
$$

where

$$
A^{\prime \prime}=\left(\begin{array}{cc}
1 & 0 \\
0 & q^{\alpha}
\end{array}\right) C A^{\prime} C^{-1}\left(\begin{array}{cc}
1 & 0 \\
0 & 1 / q^{\alpha}
\end{array}\right)=\left(\begin{array}{ll}
a^{\prime \prime} & b^{\prime \prime} \\
c^{\prime \prime} & d^{\prime \prime}
\end{array}\right) \in \Gamma_{0}(4 N) .
$$

From this we deduce that for $f \in S_{k / 2}(4 N, \psi)$

$$
f\left|W_{q}\right| A^{\prime *}=\chi_{q}^{\alpha}\left(d^{\prime \prime}\right) f\left|A^{\prime \prime *}\right| W_{q}=\chi_{q}^{\alpha}\left(d^{\prime \prime}\right) \psi\left(d^{\prime \prime}\right) f \mid W_{q}
$$

Now, $d^{\prime \prime} \equiv q^{\alpha} a d^{\prime}-4 N c b a^{\prime} / q^{\alpha}(\bmod 4 N), q^{\alpha} a \equiv 1\left(\bmod 4 N / q^{\alpha}\right)$, and $-4 N c b / q^{\alpha} \equiv 1\left(\bmod q^{\alpha}\right)$, so $d^{\prime \prime} \equiv d^{\prime}(\bmod M)$ and $d^{\prime \prime} \equiv a^{\prime}$ $\left(\bmod q^{\alpha}\right)$, so

$$
\chi_{q}^{\alpha}\left(d^{\prime \prime}\right) \psi\left(d^{\prime \prime}\right)=\chi_{q}^{\alpha}\left(d^{\prime}\right) \bar{\psi}_{Q}\left(d^{\prime}\right) \psi_{M}\left(d^{\prime}\right)
$$

which completes the proof.

The next several propositions are analogous to results obtained for integral weight forms by $\mathrm{Li}[7]$.

Proposition 3.4. Suppose $q, q^{\prime}$ are distinct primes dividing $N$, and that $\operatorname{ord}_{q} N=1$. Then $B_{q^{\prime}} W_{q}^{4 N}=W_{q}^{4 N / q^{\prime}} B_{q^{\prime}}$.

Proof. Let $C=\left(\begin{array}{cc}q a & b \\ 4 N c / q & 1\end{array}\right) \in \Gamma_{1}(4 N / q)$. Then using our previous techniques we find that

$$
\left[\left(\begin{array}{ll}
q^{\prime} & 0 \\
0 & 1
\end{array}\right),\left(q^{\prime}\right)^{-1 / 4}\right] C^{*}\left[\left(\begin{array}{cc}
1 / q^{\prime} & 0 \\
0 & 1
\end{array}\right),\left(q^{\prime}\right)^{1 / 4}\right]=\chi_{q^{\prime}}(1) C^{\prime *}=C^{\prime *}
$$


where $C^{\prime}=\left(\begin{array}{ll}q^{\prime} & 0 \\ 0 & 1\end{array}\right) C\left(\begin{array}{cc}1 / q^{\prime} & 0 \\ 0 & 1\end{array}\right)$. Since diagonal matrices commute, it follows that

$$
B_{q^{\prime}} W_{q}^{4 N} B_{q^{\prime}}^{-1}=W_{q}^{4 N / q^{\prime}} .
$$

Proposition 3.5. Consider $S_{k / 2}(4 N, \psi)$ and suppose that $q$ is a prime such that $\operatorname{ord}_{q} N=1$ and $\psi \chi_{q}$ is defined modulo $4 N / q$. Then $T_{q}+q^{k / 4-1} W_{q}$ maps $S_{k / 2}(4 N, \psi)$ to $S_{k / 2}\left(4 N / q, \psi \chi_{q}\right)$.

Proof. Recall that for $f \in S_{k / 2}(4 N, \psi), f\left|T_{q}=q^{k / 4-1} f\right| \xi \mid \sum_{k} A_{k}^{*}$ where $\xi=\left[\left(\begin{array}{ll}1 & 0 \\ 0 & q\end{array}\right), q^{1 / 4}\right] \in \mathcal{G}$ and where $\left\{A_{k}^{*}\right\}$ is a complete set of coset representatives for

$$
\left(\xi^{-1} \Gamma_{1}(4 N)^{*} \xi \cap \Gamma_{1}(4 N)^{*}\right) \backslash \Gamma_{1}(4 N)^{*} .
$$

One checks that

$$
\left(\xi^{-1} \Gamma_{1}(4 N)^{*} \xi \cap \Gamma_{1}(4 N)^{*}\right)=\left(\Gamma_{1}(4 N) \cap \Gamma_{0}(4 N, q)\right)^{*},
$$

so one may take $A_{k}=\left(\begin{array}{ll}1 & k \\ 0 & 1\end{array}\right), k=0, \ldots, q-1$. From the definition, $f\left|W_{q}=\left(\psi \chi_{q}\right)_{q}(b) f\right| \xi \mid C^{*}$ with $C \in \Gamma_{1}(4 N / q)$ of the required form. The hypothesis about the character and the restrictions on the level require that the $q$-part of $\psi$ equal $\chi_{q}$, hence $\left(\psi \chi_{q}\right)_{q}(b)=1$. For notational ease, we put $A_{q}=C$, thus

$$
f\left|\left(T_{q}+q^{k / 4-1} W_{q}\right)=q^{k / 4-1} f\right| \xi \mid \sum_{k=0}^{q} A_{k}^{*} .
$$

Let $A=\left(\begin{array}{ll}a & b \\ c & d\end{array}\right) \in \Gamma_{0}(4 N)$. As in Lemma 3 of [7], we see that

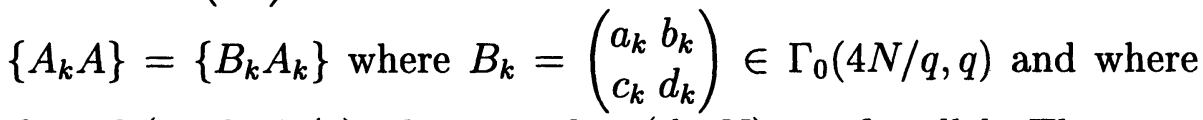
$d_{k} \equiv d(\bmod 4 N / q) ;$ also note that $\left(d_{k}, N\right)=1$ for all $k$. Thus

$$
\begin{aligned}
f\left|\left(T_{q}+q^{k / 4-1} W_{q}\right)\right| A^{*} & =q^{k / 4-1} f|\xi| \sum_{k=0}^{q} B_{k}^{*} A_{k}^{*} \\
& =q^{k / 4-1} f \mid \sum_{k=0}^{q} \xi B_{k}^{*} \xi^{-1} \xi A_{k}^{*} .
\end{aligned}
$$


Now $\xi B_{k}^{*} \xi^{-1}=\chi_{q}\left(d_{k}\right) C_{k}^{*}$ where $C_{k}=\left(\begin{array}{ll}1 & 0 \\ 0 & q\end{array}\right) B_{k}\left(\begin{array}{ll}1 & 0 \\ 0 & q\end{array}\right)^{-1} \in \Gamma_{0}(4 N)$. Thus

$$
\begin{aligned}
f\left|\left(T_{q}+q^{k / 4-1} W_{q}\right)\right| A^{*} & =q^{k / 4-1} \sum_{k=0}^{q} \chi_{q}\left(d_{k}\right) f\left|C_{k}^{*}\right| \xi A_{k}^{*} \\
& =\sum_{k=0}^{q} \chi_{q}\left(d_{k}\right) \psi\left(d_{k}\right) f \mid \xi A_{k}^{*} .
\end{aligned}
$$

Since $\left(d_{k}, N\right)=1, \chi_{q}\left(d_{k}\right) \psi\left(d_{k}\right)=\psi \chi_{q}\left(d_{k}\right)$ and since $\psi \chi_{q}$ is defined modulo $4 N / q$ and $d_{k} \equiv d(\bmod 4 N / q)$, we have

$$
\begin{aligned}
f\left|\left(T_{q}+q^{k / 4-1} W_{q}\right)\right| A^{*} & =q^{k / 4-1} \psi \chi_{q}(d) f \mid \sum_{k=0}^{q} \xi A_{k}^{*} \\
& =\psi \chi_{q}(d) f \mid\left(T_{q}+q^{k / 4-1} W_{q}\right)
\end{aligned}
$$

which completes the proof.

Proposition 3.6. Suppose $\ell$ is a prime (not necessarily dividing $N)$ and $f=g \mid B_{\ell} \in S_{k / 2}(4 N, \psi)$ with $g(\tau)=\sum_{n \geq 1} a(n) e^{2 \pi i n \tau}$. If $\ell \mid N$ and $\psi \chi_{\ell}$ is a character modulo $4 N / \ell$ then $g \in S_{k / 2}\left(4 N / \ell, \psi \chi_{\ell}\right)$; otherwise $g=0$ (and hence $f=0)$.

$$
\text { Proof. } g\left|B_{\ell}=\ell^{-k / 4} g\right| \xi \text { with } \xi=\left[\left(\begin{array}{ll}
\ell & 0 \\
0 & 1
\end{array}\right), \ell^{-1 / 4}\right] \in \mathcal{G} \text {, so } f=g \mid B_{\ell}
$$
implies $g=\ell^{k / 4} f \mid \xi^{-1}$. Let $N^{\prime}=N / \ell$ or $N$ as $\ell \mid N$ or not. If $A=\left(\begin{array}{ll}a & b \\ c & d\end{array}\right) \in \Gamma_{0}\left(4 N^{\prime}, \ell\right)$, then $B=\left(\begin{array}{ll}\ell & 0 \\ 0 & 1\end{array}\right)^{-1} A\left(\begin{array}{ll}\ell & 0 \\ 0 & 1\end{array}\right) \in \Gamma_{0}(4 N)$ and $\xi^{-1} A^{*} \xi=\chi_{\ell}(d) B^{*}$. Thus

$$
\begin{gathered}
g\left|A^{*}=\ell^{k / 4} f\right| \xi^{-1}\left|A^{*}=\ell^{k / 4} f\right| \xi^{-1} \mid A^{*} \xi \xi^{-1} \\
\ell^{k / 4} \chi_{\ell}(d) f\left|B^{*}\right| \xi^{-1}=\ell^{k / 4} \psi(d) \chi_{\ell}(d) f \mid \xi^{-1}=\psi \chi_{\ell}(d) g .
\end{gathered}
$$

Moreover, the Fourier expansion of $g$ implies that $g \mid\left(\begin{array}{ll}1 & m \\ 0 & 1\end{array}\right)^{*}=g$ for any integer $m$.

Case I. $\ell \mid N$ and $\psi \chi_{\ell}$ is defined modulo $4 N / \ell=4 N^{\prime}$. 
Any matrix $A=\left(\begin{array}{ll}a & b \\ c & d\end{array}\right) \in \Gamma_{0}\left(4 N^{\prime}\right)$ can be written in the form $\left(\begin{array}{ll}1 & m \\ 0 & 1\end{array}\right) A^{\prime}\left(\begin{array}{ll}1 & n \\ 0 & 1\end{array}\right)$ for some integers $m$ and $n$ and $A^{\prime}=\left(\begin{array}{ll}a^{\prime} & b^{\prime} \\ c^{\prime} & d^{\prime}\end{array}\right) \in$ $\Gamma_{0}\left(4 N^{\prime}, \ell\right)$. Combining the observations above, we see that $g \mid A^{*}=$ $g \mid\left(\begin{array}{ll}1 & m \\ 0 & 1\end{array}\right)^{*} A^{\prime *}\left(\begin{array}{ll}1 & n \\ 0 & 1\end{array}\right)^{*}=\psi \chi_{\ell}\left(d^{\prime}\right) g$. Since $d \equiv d^{\prime}\left(\bmod 4 N^{\prime}\right)$ and $\psi \chi_{\ell}$ is defined modulo $4 N^{\prime}$, we have $\psi \chi_{\ell}(d)=\psi \chi_{\ell}\left(d^{\prime}\right)$ which completes the proof of this case.

Case II. $\ell \nmid N$, or $\ell \mid N$ and $\psi \chi_{\ell}$ not defined modulo $4 N^{\prime}=4 N / \ell$.

In either case it is trivial to check that $\operatorname{cond}\left(\psi \chi_{\ell}\right)$ divides $4 N^{\prime} \ell$, but not $4 N^{\prime}$. Thus there exists an integer $v \not \equiv 0(\bmod \ell)$ with $(1+$ $\left.4 N^{\prime} v, \operatorname{cond}\left(\psi \chi_{\ell}\right)\right)=1$ and $\psi \chi_{\ell}\left(1+4 N^{\prime} v\right) \neq 1$. Put $A=\left(\begin{array}{cc}1 & 0 \\ 4 N^{\prime} v & 1\end{array}\right)$ and note that $A \in \Gamma_{0}\left(4 N^{\prime}, \ell\right)$. Since $1+4 N^{\prime} v \not \equiv 0(\bmod \ell)$, we may choose an integer $u$ such that $u\left(1+4 N^{\prime} v\right)+1 \equiv 0(\bmod \ell)$. Then

$$
\begin{aligned}
A^{\prime} & =\left(\begin{array}{ll}
1 & u \\
0 & 1
\end{array}\right) A\left(\begin{array}{ll}
1 & 1 \\
0 & 1
\end{array}\right) \\
& =\left(\begin{array}{cc}
1+4 N^{\prime} u v & u\left(1+4 N^{\prime} v\right)+1 \\
4 N^{\prime} v & 1+4 N^{\prime} v
\end{array}\right) \in \Gamma_{0}\left(4 N^{\prime}, \ell\right),
\end{aligned}
$$

so by the earlier observations

$$
\begin{aligned}
\psi \chi_{\ell}\left(1+4 N^{\prime} v\right) g & =g\left|A^{\prime *}=g\right|\left(\begin{array}{ll}
1 & u \\
0 & 1
\end{array}\right)^{*} A^{*}\left(\begin{array}{ll}
1 & 1 \\
0 & 1
\end{array}\right)^{*} \\
& =g\left|A^{*}\left(\begin{array}{ll}
1 & 1 \\
0 & 1
\end{array}\right)^{*}=\psi \chi_{\ell}(1) g\right|\left(\begin{array}{ll}
1 & 1 \\
0 & 1
\end{array}\right)^{*}=\psi \chi_{\ell}(1) g .
\end{aligned}
$$

Since $\psi \chi_{\ell}\left(1+4 N^{\prime} v\right) \neq \psi \chi_{\ell}(1)$, we must have $g=0$.

Now we can prove

Proposition 3.7. Suppose $f=\sum_{n>1} a(n) e^{2 \pi i n \tau}$ is an element of $S_{k / 2}(4 N, \psi)$ such that $a(n)=0$ if $(n, \bar{K})=1$ for some fixed integer $K$. Then:

(1) $a(n)=0$ if $(n, N)=1$;

(2) if $q$ is a prime dividing $N$ and $\psi \chi_{q}$ is defined modulo $4 N / q$, 
set

$$
\varphi= \begin{cases}f \mid T_{q} & \text { if } q^{2} \mid N \text { or }(K, N)=q \\ \left(1+q^{-1}\right)^{-1}\left[f \mid T_{q}+q^{k / 4-1} W_{q}\right] & \text { otherwise }\end{cases}
$$

then $\varphi \in S_{k / 2}\left(4 N / q, \psi \chi_{q}\right)$ and $f-\left(\varphi \mid B_{q}\right)=\sum_{n \geq 1} b(n) e^{2 \pi i n \tau} \in$ $S_{k / 2}(4 N, \psi)$ with $b(n)=0$ for $(n,(K, N))=1$ or $n$ a power of $q$.

Proof. For a prime $\ell$ define the annihilator $A_{\ell}: S_{k / 2}(4 N, \psi) \rightarrow$ $S_{k / 2}\left(4 N \ell^{2}, \psi\right)$ by

$$
f\left|A_{\ell}=f-f\right| T_{\ell}^{4 N^{\prime}} \mid B_{\ell}
$$

where $N^{\prime}=\operatorname{lcm}(N, \ell)$. By Proposition 3.2, $A_{\ell} \operatorname{maps} S_{k / 2}(4 N, \psi)$ into $S_{k / 2}(4 N \ell, \psi)$ if $\ell \mid N$, and $A_{\ell}$ maps $S_{k / 2}(4 N, \psi)$ into $S_{k / 2}(4 N, \psi)$ if $\ell^{2} \mid N$ and $\psi \chi_{\ell}$ is defined modulo $4 N / \ell$. Let $\ell_{1}, \ldots, \ell_{r}$ be the distinct primes dividing $K$, and suppose $\ell_{r}$ does not divide $N$. By hypothesis, the $n^{\text {th }}$ Fourier coefficient of $f\left|A_{\ell_{1}}\right| A_{\ell_{2}}|\cdots| A_{\ell_{r-1}}$ is nonzero only when $\ell_{r} \mid n$. Hence $f\left|A_{\ell_{1}}\right| \cdots\left|A_{\ell_{r-1}}=\Phi\right| B_{\ell_{r}}$ where $\Phi=\sum_{n \geq 1} c(n) e^{2 \pi i n \tau}$. Applying Proposition 3.6, we see that $\Phi$ must be 0 ; hence $a(n)=0$ when $\left(n, \ell_{1} \cdots \ell_{r-1}\right)=1$. Arguing by induction on $r$ shows that $a(n)=0$ when $(n, N)=1$. Since $(n, N)=1$ or $(n, K)=1$ implies $a(n)=0,(n,(K, N))=1$ also implies $a(n)=0$.

Now, suppose that $q$ is a prime dividing $N$ such that $\psi \chi_{q}$ is defined modulo $4 N / q$. If $q^{2} \mid N$ then the theorem follows from Proposition 3.2. If $(K, N)=q$ then by Proposition $3.6 f=g \mid B_{q}$ with $g \in S_{k / 2}\left(4 N, \psi \chi_{q}\right)$, and thus $\varphi=g\left|B_{q}\right| T_{q}=g$ and $f-\left(\varphi \mid B_{q}\right)=0$. Finally we consider the case where $\operatorname{ord}_{q}(N)=1$ and $(K, N) \neq$ q. By Proposition 3.5, $\varphi=\left(1+q^{-1}\right)^{-1} f \mid\left(T_{q}+q^{k / 4-1} W_{q}\right) \in$ $S_{k / 2}\left(4 N / q, \psi \chi_{q}\right)$. In particular, we note that the $q$-part of the character $\psi \chi_{q}$ is trivial. We have already established that $a(n)=0$ for $(n,(K, N))=1$. If $(K, N)=1$, the result is obvious. Let $q_{1}, \ldots, q_{t}=q$ be the distinct primes dividing $(K, N) ;$ since $(K, N) \neq$ $q$ and $\operatorname{ord}_{q}(N)=1$, we must have $t>1$. Looking at the Fourier coefficients of $f$, we see that $f=\sum_{j=1}^{t} \Phi_{j} \mid B_{q_{j}}$ where $\Phi_{1}=q_{1}^{k / 4-1} f \mid T_{q_{1}}$, and $\Phi_{j}=q_{j}^{k / 4-1} f\left|A_{q_{1}}\right| \cdots\left|A_{q_{j-1}}\right| T_{q_{j}}$ for $j>1$. Clearly $\Phi_{j} \mid B_{q_{j}} \in S_{k / 2}\left(4 N q_{1}^{2} \cdots q_{j}^{2}, \psi\right)$ for all $j$. Also,

$$
\begin{aligned}
\Phi_{t} \mid B_{q_{t}} & =\left(\sum_{j=1}^{t} \Phi_{j} \mid B_{q_{j}}\right)\left|A_{q_{1}}\right| \cdots \mid A_{q_{t-1}} \\
& =f\left|A_{q_{1}}\right| \cdots \mid A_{q_{t-1}} \in S_{k / 2}\left(4 N^{\prime}, \psi\right)
\end{aligned}
$$


where $N^{\prime}=N q_{1}^{2} \cdots q_{t-1}^{2}$, so by Proposition 3.6,

$$
\Phi_{t} \in S_{k / 2}\left(4 N q_{1}^{2} \cdots q_{t-1}^{2} / q, \psi \chi_{q}\right) .
$$

Now,

$$
\begin{aligned}
& \Phi_{t}\left|B_{q}\right|\left(T_{q}+q^{k / 4-1} W_{q}\right) \\
&=\Phi_{t}+q^{k / 4-1} \Phi_{t}\left|B_{q}\right| W_{q} \\
&=\Phi_{t}+q^{k / 4-1} q^{-k / 4}\left(\psi \chi_{q}\right)_{q}(b) \cdot \\
& \Phi_{t}\left|\left[\left(\begin{array}{ll}
q & 0 \\
0 & 1
\end{array}\right), q^{-1 / 4}\right]\right|\left[\left(\begin{array}{ll}
1 & 0 \\
0 & q
\end{array}\right), q^{1 / 4}\right]\left(\begin{array}{ll}
a & b \\
c & d
\end{array}\right)^{*} \\
&=\left(1+q^{-1}\right) \Phi_{t}
\end{aligned}
$$

where $\left(\begin{array}{ll}a & b \\ c & d\end{array}\right) \in \Gamma_{1}\left(4 N q_{1}^{2} \cdots q_{t-1}^{2} / q\right)$ is chosen to represent $W_{q}$ and where we recall that the $q$-part of $\psi \chi_{q}$ is trivial. For $1 \leq j<t$, Proposition 3.4 shows that

$$
\Phi_{j}\left|B_{q_{j}}\right|\left(T_{q}+q^{k / 4-1} W_{q}\right)=\Phi_{j}\left|T_{q}\right| B_{q_{j}}+q^{k / 4-1} \Phi_{j}\left|W_{q}^{4 N^{\prime} / q_{j}}\right| B_{q_{j}},
$$

and $\left(\Phi_{j} \mid T_{q}\right)+q^{k / 4-1}\left(\Phi_{j} \mid W_{q}^{4 N^{\prime} / q_{j}}\right) \in S_{k / 2}\left(4 N^{3}, \psi \chi_{q} \chi_{q_{j}}\right)$. Therefore

$$
\begin{aligned}
\left(1+q^{-1}\right) \varphi & =\sum_{j=1}^{t} \Phi_{j}\left|B_{q_{j}}\right|\left(T_{q}+q^{k / 4-1} W_{q}^{4 N^{\prime}}\right) \\
& =\left(1+q^{-1}\right) \Phi_{t}+\sum_{j=1}^{t-1} \Phi_{j}\left|\left(T_{q}+q^{k / 4-1} W_{q}^{4 N^{\prime} / q_{j}}\right)\right| B_{q_{j}}
\end{aligned}
$$

and so

$$
\begin{aligned}
f-\varphi & \mid B_{q} \\
= & \left(\sum_{j=1}^{t} \Phi_{j} \mid B_{q_{j}}\right) \\
& -\left(\Phi_{t} \mid B_{q}\right)-\left(1+q^{-1}\right)^{-1} \sum_{j=1}^{t-1} \Phi_{j}\left|\left(T_{q}+q^{\frac{k}{4}-1} W_{q}^{4 N^{\prime} / q_{j}}\right)\right| B_{q_{j}} \mid B_{q} \\
& =\sum_{j=1}^{t-1}\left(\Phi_{j}-\left(1+q^{-1}\right)^{-1} \Phi_{j}\left|\left(T_{q}+q^{\frac{k}{4}-1} W_{q}^{4 N^{\prime} / q_{j}}\right)\right| B_{q}\right) \mid B_{q_{j}}
\end{aligned}
$$


Thus if $\left(n, q_{1} \cdots q_{t-1}\right)=1$ or $n$ is a power of $q$, then the $n^{\text {th }}$ Fourier coefficient of $f-\left(\varphi \mid B_{q}\right)$ is zero.

Now recall that the space spanned by the oldforms, $S_{k / 2}^{-}(4 N, \psi)$ is the subspace of $S_{k / 2}(4 N, \psi)$ spanned by forms and shifts of forms of lower levels, that is

$$
S_{k / 2}^{-}(4 N, \psi)=\sum_{\substack{N^{\prime} \ell \mid N \\ N^{\prime}<N}} S_{k / 2}\left(4 N^{\prime}, \psi \chi_{\ell}\right) \mid B_{\ell} .
$$

Also, the subspace spanned by the newforms, $S_{k / 2}^{+}(4 N, \psi)$, is the orthogonal complement of $S_{k / 2}^{-}(4 N, \psi)$ in $S_{k / 2}(4 N, \psi)$ with respect to the Petersson inner product (see [11] for details).

THEOREM 3.8. Suppose $f(\tau)=\sum_{n \geq 1} a(n) e^{2 \pi i n \tau} \in S_{k / 2}(4 N, \psi)$ and $a(n)=0$ whenever $(n, K)=1$ for some fixed integer $K$. Then $f \in S_{k / 2}^{-}(4 N, \psi)$.

Proof. Let $q_{1}, \cdots, q_{r}, \cdots, q_{r+s}$ be the distinct primes dividing $(N, K)$ such that $\psi \chi_{q_{j}}$ is defined modulo $4 N / q_{j}$ if and only if $r<$ $j \leq r+s$. If $s>0$ then Proposition 3.7 says that there is some $\varphi \in$ $S_{k / 2}\left(4 N / q_{r+s}, \psi \chi_{q_{r+s}}\right)$ such that $\left(f-\varphi \mid B_{q_{r+s}}\right)(\tau)=\sum_{n \geq 1} b(n) e^{2 \pi i n \tau}$ where $b(n) \neq 0$ only if $\left(n, q_{1} \cdots q_{r+s-1}\right)>1$. Thus induction on $s$ shows that $f=h+g$ where $h \in S_{k / 2}^{-}(4 N, \psi)$ and $g(\tau)=$ $\sum_{n \geq 1} c(n) e^{2 \pi i n \tau}$ where $c(n) \neq 0$ only if $\left(n, q_{1} \cdots q_{r}\right)>1$. Then with $A_{q_{j}}$ defined as in the proof of Proposition 3.7,

$$
\begin{aligned}
g \mid A_{q_{1}} \cdots A_{q_{r-1}} & =\sum_{n \geq 1} c\left(q_{r} n\right) e^{2 \pi i q_{r} n \tau} \\
& =\sum_{n \geq 1} c\left(q_{r} n\right) e^{2 \pi i n \tau} \mid B_{q_{r}} \in S_{k / 2}\left(4 N^{\prime}, \psi\right)
\end{aligned}
$$

where $N^{\prime}=N q_{1}^{2} \cdots q_{r-1}^{2}$; since $\psi \chi_{q_{r}}$ is not defined modulo $4 N^{\prime} / q_{r}$, Proposition 3.6 shows that $g \mid A_{q_{1}} \cdots A_{q_{r-1}}=0$ and hence $c(n) \neq 0$ only if $\left(n, q_{1} \cdots q_{r-1}\right)>1$. Induction on $r$ now yields the theorem.

This allows us to prove a multiplicity-one theorem for half-integral weight newforms.

THEOREM 3.9. Suppose $f(\tau)=\sum_{n \geq 1} a(n) e^{2 \pi i n \tau}$ and $g(\tau)=$ $\sum_{n \geq 1} b(n) e^{2 \pi i n \tau}$ are newforms in $S_{k / 2}(4 N, \psi)$ such that

(1) for almost all primes $p \nmid 4 N, f \mid T_{p^{2}}=\lambda_{p} f$ and $g \mid T_{p^{2}}=\lambda_{p} g$; and 
(2) for almost all squarefree integers $t, a(t)=b(t)$.

Then $f=g$.

Proof. Let $K$ be the product of all primes $p$ not dividing $4 N$ such that condition (1) fails and all squarefree integers $t$ such that condition (2) fails. For $n \in \mathbb{Z}_{+}$such that $(n, 4 N K)=1$, write $n=t m^{2}$ where $t$ is squarefree; then using induction on the number of prime factors of $m$, the action of the Hecke operators on Fourier coefficients implies that $a(n)=b(n)$. Hence

$$
(f-g)(\tau)=\sum_{n \geq 1} c(n) e^{2 \pi i n \tau}
$$

with $c(n)=0$ whenever $(n, 4 N K)=1$, and so $f-g \in S_{k / 2}^{-}(4 N, \psi)$ by Theorem 3.8. Since $f, g \in S_{k / 2}^{+}(4 N, \psi)$, we must have $f=g$.

4. The Hilbert Modular Case. In this section, we describe generalizations of the results in $\S 3$ to the Hilbert modular setting. In general, the proofs involved are analogous to those in the elliptic case, so we focus solely on developing the necessary operators and their properties.

Let $K$ be a totally real number field of degree $n$ over $\mathbb{Q}$ with ring of integers $\mathcal{O}$, unit group $\mathcal{U}$, totally positive units $\mathcal{U}^{+}$, and group of square units $\mathcal{U}^{2}$. Let $\mathcal{N}$ be an integral ideal and $\mathcal{I}$ a fractional ideal; define

$$
\begin{gathered}
\Gamma_{0}(\mathcal{N}, \mathcal{I})=\left\{A \in\left(\begin{array}{cc}
\mathcal{O} & \mathcal{I}^{-1} \partial^{-1} \\
\mathcal{N} \mathcal{I} \partial & \mathcal{O}
\end{array}\right): \operatorname{det} A \in \mathcal{U}^{2}\right\} \\
\Gamma_{1}(\mathcal{N}, \mathcal{I})=\left\{A=\left(\begin{array}{ll}
a & b \\
c & d
\end{array}\right) \in \Gamma_{0}(\mathcal{N}, \mathcal{I}):\right. \\
\left.a \equiv u^{2}(\bmod \mathcal{N}) \text { for some } u \in \mathcal{U}\right\},
\end{gathered}
$$

and define

$$
\theta(\mathcal{I}, \tau)=\sum_{x \in \mathcal{I}} \mathrm{e}\left(2 x^{2} \tau\right)
$$

where $\tau=\left(\ldots, \tau_{j}, \ldots\right) \in \mathcal{H}^{n}$ and for $\alpha \in K, \operatorname{Tr}(\alpha \tau)=\sum_{j} \alpha^{(j)} \tau_{j}$ $\left(\alpha^{(j)}\right.$ the conjugates of $\alpha$ over $\left.\mathbb{Q}\right)$ and $\mathrm{e}(\alpha \tau)=e^{\pi i \operatorname{Tr}(\alpha \tau)}$. Let $\mathcal{G}$ denote 
the group extension of $\mathrm{GL}_{2}^{+}(K)$ consisting of pairs $[A, \varphi(\tau)]$ where $A=\left(\begin{array}{ll}a & b \\ c & d\end{array}\right) \in \mathrm{GL}_{2}^{+}(K)$ and where $\varphi(\tau)$ is a holomorphic function on $\mathcal{H}^{n}$ satisfying $\varphi(\tau)^{2}=t N(c \tau+d) N(\operatorname{det} A)^{-1 / 2}$ with $|t|=1$. We refer to the function $\varphi$ as a factor of automorphy for the matrix $A$. The group law in $\mathcal{G}$ is given by $[A, \varphi(\tau)][B, \psi(\tau)]=[A B, \varphi(B \tau) \psi(\tau)]$. For $A \in \Gamma_{0}\left(4 \mathcal{O}, \mathcal{I}^{2}\right), j_{\mathcal{I}}(A, \tau)=\theta(\mathcal{I}, A \tau) / \theta(\mathcal{I}, \tau)$ is a well-defined automorphy factor for $A$ (see [21] and [23]); moreover, $j_{\mathcal{I}}(A, \tau)=$ $j_{\mathcal{J}}(A, \tau)$ whenever $A \in \Gamma_{0}\left(4 \mathcal{O}, \mathcal{I}^{2}\right) \cap \Gamma_{0}\left(4 \mathcal{O}, \mathcal{J}^{2}\right)$.

For $A \in \Gamma_{0}\left(4 \mathcal{O}, \mathcal{I}^{2}\right)$, put $A^{*}=\left[A, j_{\mathcal{I}}(\tau)\right] \in \mathcal{G}$, and for any subgroup $\Gamma \subset \Gamma_{0}\left(4 \mathcal{O}, \mathcal{I}^{2}\right)$ define $\Gamma^{*}=\left\{A^{*}: A \in \Gamma\right\}$ which is a subgroup of $\mathcal{G}$. Finally for a holomorphic function $f$ on $\mathcal{H}^{n}$ and $[A, \varphi(\tau)] \in \mathcal{G}$, define the slash operator by

$$
\left.f\right|_{k / 2}[A, \varphi(\tau)]=f \mid[A, \varphi(\tau)]=\varphi(\tau)^{-k} f(A \tau) .
$$

Next, let $\psi$ be a numerical character modulo $4 \mathcal{N}$ and $k$ an odd rational integer; let $\mathcal{M}_{k / 2}\left(\Gamma_{0}\left(4 \mathcal{N}, \mathcal{I}^{2}\right)^{*}, \psi\right)$ denote the space of Hilbert modular forms of (uniform) weight $k / 2$ and character $\psi$ for the group $\Gamma_{0}\left(4 \mathcal{N}, \mathcal{I}^{2}\right)^{*}$, that is the set of functions $f$ which are holomorphic on $\mathcal{H}^{n}$ and at the cusps satisfying $f(A \tau)=\psi(a) j_{\mathcal{I}}(A, \tau) f(\tau)$. As shown in $\S 2$ of $[\mathbf{2 3}], \mathcal{M}_{k / 2}\left(\Gamma_{0}\left(4 \mathcal{N}, \mathcal{I}^{2}\right)^{*}, \psi\right)=\{0\}$ unless $\psi(u)=1$ for all $u \in \mathcal{U}$, so we will assume throughout that $\psi$ is trivial on $\mathcal{U}$.

For the convenience of the reader we briefly recall the definition of two operators whose definitions and properties appear in [23]. Fix a space of modular forms $\mathcal{M}_{k / 2}\left(\Gamma_{0}\left(4 \mathcal{N}, \mathcal{I}^{2}\right)^{*}, \psi\right)$. For a prime $\mathcal{P} \nmid 4 \mathcal{N}$, let $\left\{A_{j}^{*}\right\}$ denote a complete set of representatives for

$$
\left(\Gamma_{1}\left(4 \mathcal{N}, \mathcal{I}^{2}\right)^{*} \cap \Gamma_{1}\left(4 \mathcal{N}, \mathcal{P}^{2} \mathcal{I}^{2}\right)^{*}\right) \backslash \Gamma_{1}\left(4 \mathcal{N}, \mathcal{P}^{2} \mathcal{I}^{2}\right)^{*}
$$

The Hecke operator $T_{\mathcal{P}^{2}}$ maps $\mathcal{M}_{k / 2}\left(\Gamma_{0}\left(4 \mathcal{N}, \mathcal{I}^{2}\right)^{*}, \psi\right)$ to $\mathcal{M}_{k / 2}\left(\Gamma_{0}\left(4 \mathcal{N}, \mathcal{P}^{2} \mathcal{I}^{2}\right)^{*}, \psi\right)$ and is defined by

$$
f \mapsto f\left|T_{\mathcal{P}^{2}}=N(\mathcal{P})^{k / 2-2} \sum_{j} f\right| A_{j}^{*} .
$$

Analogous to the integral weight case, we have an operator $S_{\mathcal{P}}$ which also maps the space $\mathcal{M}_{k / 2}\left(\Gamma_{0}\left(4 \mathcal{N}, \mathcal{I}^{2}\right)^{*}, \psi\right)$ to $\mathcal{M}_{k / 2}\left(\Gamma_{0}\left(4 \mathcal{N}, \mathcal{P}^{2} \mathcal{I}^{2}\right)^{*}, \psi\right)$ defined by

$$
f\left|S_{\mathcal{P}}=f\right|\left[C, N(\mathcal{P})^{-1 / 2} \frac{\theta(\mathcal{I}, C \tau)}{\theta(\mathcal{P} \mathcal{I}, \tau)}\right]
$$


where

$$
C \in\left(\begin{array}{cc}
\mathcal{P} & \mathcal{P}^{-1} \mathcal{I}^{-2} \partial^{-1} \\
\mathcal{N} \mathcal{P} \mathcal{I}^{2} \partial & \mathcal{O}
\end{array}\right), \operatorname{det} C=1, \text { and } a_{C} \equiv 1 \quad(\bmod \mathcal{N})
$$

In fact, $S_{\mathcal{P}}$ is an isomorphism, so by setting $S_{\mathcal{P}^{-1}}=S_{\mathcal{P}}^{-1}$ and $S_{\mathcal{J}_{1}} S_{\mathcal{J}_{2}}=S_{\mathcal{J}_{1} \mathcal{J}_{2}}$, we can inductively define $S_{\mathcal{J}}$ for any fractional ideal $\mathcal{J}$ relatively prime to $\mathcal{N}$. The relationship between the operators $T_{\mathcal{P}^{2}}$ and $S_{\mathcal{P}}$ is given by Lemma 2.2 of [23] which we restate below.

LEMMA 4.1. For $\mathcal{P}$ a prime, $\mathcal{P} \nmid 4 \mathcal{N}$, and $f \in \mathcal{M}_{k / 2}\left(\Gamma_{0}\left(4 \mathcal{N}, \mathcal{I}^{2}\right)^{*}, \psi\right)$ we have

$$
\begin{aligned}
& N(\mathcal{P})^{2-\frac{k}{2}} f\left|T_{\mathcal{P}^{2}}=\sum_{b} f\right|\left[\left(\begin{array}{ll}
1 & b \\
0 & 1
\end{array}\right), 1\right] \\
& +\sum_{\beta} f\left|S_{\mathcal{P}}\right|\left[\left(\begin{array}{ll}
1 & \beta \\
0 & 1
\end{array}\right), N(\mathcal{P})^{\frac{1}{2}}\left(\sum_{\alpha \in \mathcal{P} \mathcal{I} / \mathcal{P}^{2} \mathcal{I}} e\left(-2 \beta \alpha^{2}\right)\right)^{-1}\right] \\
& \quad+f \mid S_{\mathcal{P}^{2}}
\end{aligned}
$$

where $b$ runs over $\mathcal{P}^{-2}\left(\mathcal{I}^{2} \partial\right)^{-1} /\left(\mathcal{I}^{2} \partial\right)^{-1}$ and $\beta$ runs over

$$
\left(\mathcal{P}^{-1}\left(\mathcal{P}^{2} \mathcal{I}^{2} \partial\right)^{-1} /\left(\mathcal{P}^{2} \mathcal{I}^{2} \partial\right)^{-1}\right)^{\times}
$$

Let $\mathcal{I}_{1}, \ldots, \mathcal{I}_{h^{\prime}}$ be ideals representing the (nonstrict) ideal classes of $K$; then by the Global Square Theorem, $\mathcal{I}_{1}^{2}, \ldots, \mathcal{I}_{h^{\prime}}^{2}$ lie in distinct strict ideal classes. Let

$$
\mathcal{M}_{k / 2}(4 \mathcal{N}, \psi)=\prod_{\lambda=1}^{h^{\prime}} \mathcal{M}_{k / 2}\left(\Gamma_{0}\left(4 \mathcal{N}, \mathcal{I}_{\lambda}^{2}\right)^{*}, \psi\right)
$$

Whenever $\mathcal{I}$ and $\mathcal{J}$ are fractional ideals in the same (nonstrict) ideal class, say $\alpha \mathcal{I}=\mathcal{J}$ with $\alpha \in K^{\times}$, the mapping $f \mapsto f \mid$ $\left[\left(\begin{array}{cc}\alpha^{-2} & 0 \\ 0 & 1\end{array}\right), N\left(\alpha^{2}\right)^{1 / 4}\right]$ is an isomorphism from the space $\mathcal{M}_{k / 2}\left(\Gamma_{0}\left(4 \mathcal{N}, \mathcal{I}^{2}\right)^{*}, \psi\right)$ onto $\mathcal{M}_{k / 2}\left(\Gamma_{0}\left(4 \mathcal{N}, \mathcal{J}^{2}\right)^{*}, \psi\right)$; notice that this isomorphism is independent of the choice of $\alpha$. Hence by considering 
the action componentwise, we can consider $T_{\mathcal{P}^{2}}$ and $S_{\mathcal{P}}$ as operators on the space $\mathcal{M}_{k / 2}(4 \mathcal{N}, \psi)$.

For any Hecke character $\Psi$ extending $\psi$ (cf. [22], [12]) there is an associated ideal character $\Psi^{*}$ whose conductor divides $4 \mathcal{N}$. By setting

$$
\begin{aligned}
& \mathcal{M}_{k / 2}(4 \mathcal{N}, \Psi)=\left\{F \in \mathcal{M}_{k / 2}(4 \mathcal{N}, \psi): F \mid S_{\mathcal{P}}\right. \\
& \left.=\Psi^{*}(\mathcal{P}) F \text { for all primes } \mathcal{P} \nmid 4 \mathcal{N}\right\},
\end{aligned}
$$

we get

$$
\mathcal{M}_{k / 2}(4 \mathcal{N}, \psi)=\oplus_{\Psi} \mathcal{M}_{k / 2}(4 \mathcal{N}, \Psi)
$$

where the sum is over all Hecke characters $\Psi$ extending $\psi$ with $\Psi_{\infty}=1$ (cf. [22], [23]).

Let $F=\left(\ldots, f_{\lambda}, \ldots\right) \in \mathcal{M}_{k / 2}(4 \mathcal{N}, \Psi)$. Notice that since $f_{\lambda} \mid$ $\left[\left(\begin{array}{ll}1 & b \\ 0 & 1\end{array}\right), 1\right]=f_{\lambda}$ for all $b \in \mathcal{I}_{\lambda}^{-2} \partial^{-1}, f_{\lambda}$ has a Fourier expansion of the form

$$
f_{\lambda}(\tau)=\sum_{\xi \in \mathcal{I}_{\lambda}^{2}} a_{\lambda}(\xi) e^{2 \pi i \operatorname{Tr}(\xi \tau)}
$$

with $\xi=0$ or $\xi \gg 0$. For $F=\left(\ldots, f_{\lambda}, \ldots\right) \in \mathcal{M}_{k / 2}(4 \mathcal{N}, \Psi)$, we use the coefficients $a_{\lambda}(\xi)$ to build "Fourier coefficients" associated to $F$ and attached to integral ideals. Mimicing the integral weight case, we will define Fourier coefficients only for integral ideals of the form $\xi \mathcal{I}_{\lambda}^{-2}$ where $\xi \in \mathcal{I}_{\lambda}$ is either zero or totally positive. Notice that all of these ideals lie in what Hecke refers to as the principal complex (see [3]), that is in the strict class of the square of some ideal. However, unlike the integral weight case, these Fourier coefficients depend not only upon the ideal, but also upon an extra parameter which we now describe; for more details see [23]. Let $G$ be the quotient group $K^{+} / \dot{K}^{2}$, the group of totally positive elements modulo squares, and let $H=\mathcal{U}^{+} \dot{K}^{2} / \dot{K}^{2} \cong \mathcal{U}^{+} / \mathcal{U}^{2}$, a subgroup of $G$. Let $\widehat{G}$ be the character group of $G$ and $H^{\perp}=\left\{\phi \in \widehat{G}:\left.\phi\right|_{H}=1\right\}$. For a character $\phi \in \widehat{G}$ and an integral ideal $\mathcal{M}=\xi \mathcal{I}_{\lambda}^{-2}$ for some $\xi \gg 0$, we define the $\mathcal{M}, \phi$-Fourier coefficient of $F, a(\mathcal{M}, \phi ; F)$, to be

$$
\mathbf{a}(\mathcal{M}, \phi ; F)=\frac{N\left(\mathcal{I}_{\lambda}\right)^{-k / 2}}{\left[\mathcal{U}^{+}: \mathcal{U}^{2}\right]} \sum_{u \in \mathcal{U}^{+} / \mathcal{U}^{2}} \bar{\phi}(\xi u) a_{\lambda}(\xi u)
$$


where $f_{\lambda}(\tau)=\sum_{\zeta} a_{\lambda}(\zeta) \mathrm{e}(2 \zeta \tau)$. Notice that $\mathbf{a}(\mathcal{M}, \phi ; F)$ depends only upon the choice of $\phi$ modulo $H^{\perp}$. If $\mathcal{M}$ is a nonzero integral ideal, but not of the form $\mathcal{M}=\xi \mathcal{I}_{\lambda}^{-2}$ for any $\lambda$, we put $\mathbf{a}(\mathcal{M}, \phi ; F)=$ 0 for all $\phi$. Finally, if $\mathcal{M}=0$ and $N\left(\mathcal{I}_{\lambda}\right)^{-k / 2} a_{\lambda}(0)=N\left(\mathcal{I}_{\mu}\right)^{-k / 2} a_{\mu}(0)$ for all $\lambda, \mu$ then we set $\mathbf{a}(0, \phi ; F)=N\left(\mathcal{I}_{\lambda}\right)^{-k / 2} a_{\lambda}(0)$. As shown in $\S 2$ of [23], the $\mathcal{M}, \phi$-Fourier coefficients of $F$ characterize $F$ whenever the $0, \phi$-Fourier coefficients of $F$ can be defined. In particular, these Fourier coefficients characterize any cusp form in $\mathcal{M}_{k / 2}(4 \mathcal{N}, \Psi)$.

Now, each space $\mathcal{M}_{k / 2}(4 \mathcal{N}, \Psi)$ is invariant under the Hecke operators $T_{\mathcal{P}^{2}}$ where $\mathcal{P}$ is a prime ideal, $\mathcal{P} \nmid 4 \mathcal{N}$, and the action of such $T_{\mathcal{P}^{2}}$ on the Fourier coefficients of $F \in \mathcal{M}_{k / 2}(4 \mathcal{N}, \Psi)$ is a natural extension of their action when $K=\mathbb{Q}$ (Theorem 2.5 of [23]). To make clear the action of $T_{\mathcal{P}^{2}}$ on the components of a modular form $F=\left(\ldots, f_{\lambda}, \ldots\right) \in \mathcal{M}_{k / 2}(4 \mathcal{N}, \Psi)$, fix an index $\lambda$ and choose an index $\mu$ and $\gamma \in K^{\times}$such that $\mathcal{P} \mathcal{I}_{\lambda}=\gamma \mathcal{I}_{\mu}$. Take $\left\{A_{j}{ }^{*}\right\}$ to be a complete set of coset representatives for

$$
\begin{aligned}
\left(\left[\left(\begin{array}{cc}
1 & 0 \\
0 & \gamma^{-2}
\end{array}\right), N\left(\gamma^{2}\right)^{-\frac{1}{4}}\right] \Gamma_{1}\left(4 \mathcal{N}, \mathcal{I}_{\lambda}^{2}\right)^{*}\right. & {\left[\left(\begin{array}{cc}
1 & 0 \\
0 & \gamma^{2}
\end{array}\right), N\left(\gamma^{2}\right)^{\frac{1}{4}}\right] } \\
& \left.\cap \Gamma_{1}\left(4 \mathcal{N}, \mathcal{I}_{\mu}^{2}\right)^{*}\right) \backslash \Gamma_{1}\left(4 \mathcal{N}, \mathcal{I}_{\mu}^{2}\right)^{*} .
\end{aligned}
$$

Then the $\mu$-component of $F \mid T_{\mathcal{P}^{2}}$ equal to

$$
\begin{aligned}
N(\mathcal{P})^{k / 2-2} f_{\lambda}\left|\left[\left(\begin{array}{cc}
1 & 0 \\
0 & \gamma^{2}
\end{array}\right), N\left(\gamma^{2}\right)^{\frac{1}{4}}\right]\right| & \\
& \sum_{J} A_{j}{ }^{*} \in \mathcal{M}_{k / 2}\left(\Gamma_{0}\left(4 \mathcal{N}, \mathcal{I}_{\mu}^{2}\right)^{*}, \psi\right) .
\end{aligned}
$$

We give an equivalent formulation of this definition which appears in [21] and from which the definiton of the Hecke operator $T_{\mathcal{Q}}$ given below will seem more natural. Choose $\rho \in K, \rho \gg 0$ such that $\mathcal{P}^{2} \mathcal{I}_{\lambda}^{2}=\rho \mathcal{I}_{\mu}^{2}$, then the representatives $\left\{A_{j}^{*}\right\}$ chosen above form a complete set of coset representatives for

$$
\begin{aligned}
\left(\left[\left(\begin{array}{cc}
1 & 0 \\
0 & \rho^{-1}
\end{array}\right), N(\rho)^{-\frac{1}{4}}\right] \Gamma_{1}\left(4 \mathcal{N}, \mathcal{I}_{\lambda}^{2}\right)^{*}\left[\left(\begin{array}{ll}
1 & 0 \\
0 & \rho
\end{array}\right), N(\rho)^{\frac{1}{4}}\right]\right. \\
\left.\cap \Gamma_{1}\left(4 \mathcal{N}, \mathcal{I}_{\mu}^{2}\right)^{*}\right) \backslash \Gamma_{1}\left(4 \mathcal{N}, \mathcal{I}_{\mu}^{2}\right)^{*}
\end{aligned}
$$


and $\rho=v \gamma^{2}$ for some $v \in \mathcal{U}^{+}$. Furthermore, letting $\phi$ vary over $\widehat{G} / H^{\perp}$ and $u$ over $\mathcal{U}^{+} / \mathcal{U}^{2}$, we have

$$
\begin{gathered}
\frac{N(\mathcal{P})^{k / 2-2}}{\left[\mathcal{U}^{+}: \mathcal{U}^{2}\right]} \sum_{\phi, u} \bar{\phi}(\rho u) f_{\lambda}\left|\left[\left(\begin{array}{cc}
1 & 0 \\
0 & \rho u
\end{array}\right), N(\rho u)^{\frac{1}{4}}\right]\right| \sum_{j} A_{j}^{*} \\
=\frac{N(\mathcal{P})^{k / 2-2}}{\left[\mathcal{U}^{+}: \mathcal{U}^{2}\right]} \sum_{\phi, u} \bar{\phi}\left(\gamma^{2} v u\right) f_{\lambda}\left|\left[\left(\begin{array}{cc}
1 & 0 \\
0 & v u
\end{array}\right), 1\right]\right| \\
\quad\left[\left(\begin{array}{cc}
1 & 0 \\
0 & \gamma^{2}
\end{array}\right), N\left(\gamma^{2}\right)^{\frac{1}{4}}\right] \mid \sum_{j} A_{j}^{*} \\
=N(\mathcal{P})^{k / 2-2} f_{\lambda}\left|\left[\left(\begin{array}{cc}
1 & 0 \\
0 & \gamma^{2}
\end{array}\right), N\left(\gamma^{2}\right)^{\frac{1}{4}}\right]\right| \sum_{j} A_{j}^{*}
\end{gathered}
$$

(since $\phi$ is quadratic and as shown in $[\mathbf{2 3}], \frac{1}{\left[\mathcal{U}^{+}: \mathcal{U}^{2}\right]} \sum_{\phi, u} \bar{\phi}(u) f_{\lambda}$ | $\left.\left[\left(\begin{array}{ll}u & 0 \\ 0 & 1\end{array}\right), 1\right]=f_{\lambda}\right)$.

We now define the operators $T_{\mathcal{Q}}, W_{\mathcal{Q}}$ (for $\mathcal{Q}$ a prime ideal dividing $\mathcal{N})$ and $B_{\mathcal{M}}$ (for $\mathcal{M}$ an integral ideal). We expect these operators to map a modular form which transforms under $\Gamma_{0}(4 \mathcal{N}, \mathcal{I})$ to a modular form which transforms under $\Gamma_{0}(4 \mathcal{N}, \mathcal{I} \mathcal{Q})$ (or $\Gamma_{0}(4 \mathcal{N}, \mathcal{I} \mathcal{M})$ ), and since we are restricting our attention to modular forms which transform under groups $\Gamma_{0}(4 \mathcal{N}, \mathcal{I})$ where $\mathcal{I}$ is in the principal ideal class complex, we shall define $T_{\mathcal{Q}}, W_{\mathcal{Q}}$ and $B_{\mathcal{M}}$ to be 0 when $\mathcal{Q}$ (or $\mathcal{M})$ is not in the principal complex.

In analogy with the above definition of $T_{\mathcal{P}^{2}}$, we define $T_{\mathcal{Q}}$ as follows. With $\mathcal{Q}$ in the principal ideal class complex, fix $\rho \gg 0$ and indices $\lambda$ and $\mu$ such that $\mathcal{Q} \mathcal{I}_{\lambda}^{2}=\rho \mathcal{I}_{\mu}^{2}$; let $\left\{A_{j}^{*}\right\}$ be a complete set of coset representatives for

$$
\begin{aligned}
\left(\left[\left(\begin{array}{cc}
1 & 0 \\
0 & \rho^{-1}
\end{array}\right), N(\rho)^{-\frac{1}{4}}\right] \Gamma_{1}\left(4 \mathcal{N}, \mathcal{I}_{\lambda}^{2}\right)^{*}\right. \\
\\
{\left.\left[\left(\begin{array}{ll}
1 & 0 \\
0 & \rho
\end{array}\right), N(\rho)^{\frac{1}{4}}\right] \cap \Gamma_{1}\left(4 \mathcal{N}, \mathcal{I}_{\mu}^{2}\right)^{*}\right) \backslash \Gamma_{1}\left(4 \mathcal{N}, \mathcal{I}_{\mu}^{2}\right)^{*} . }
\end{aligned}
$$

Set the $\mu$-component of $F \mid T_{\mathcal{Q}}$ equal to

$$
\frac{N(\mathcal{Q})^{\frac{k}{4}-1}}{\left[\mathcal{U}^{+}: \mathcal{U}^{2}\right]} \sum_{\phi, u} \bar{\phi}(\rho u) f_{\lambda}\left|\left[\left(\begin{array}{cc}
1 & 0 \\
0 & \rho u
\end{array}\right), N(\rho u)^{\frac{1}{4}}\right]\right| \sum_{j} A_{j}^{*}
$$


Clearly this definition is independent of the choice of $\rho$ and we have only one index $\mu$ corresponding to each index $\lambda$, thus $F \mid T_{\mathcal{Q}}$ is well-defined.

We define $B_{\mathcal{M}}$ in a similar fashion, although here $\mathcal{M}$ can be any integral ideal (in the principal complex). If we have $\gamma \gg 0$ such that $\mathcal{M} \mathcal{I}_{\eta}^{2}=\gamma \mathcal{I}_{\lambda}^{2}$ then we set the $\eta$-component of $F \mid B_{\mathcal{M}}$ equal to

$$
\frac{N(\mathcal{M})^{-k / 4}}{\left[\mathcal{U}^{+}: \mathcal{U}^{2}\right]} \sum_{\phi, u} \bar{\phi}(\gamma u) f_{\lambda} \mid\left[\left(\begin{array}{cc}
\gamma u & 0 \\
0 & 1
\end{array}\right), N(\gamma u)^{-\frac{1}{4}}\right] \text {. }
$$

Before defining $W_{\mathcal{Q}}=W_{\mathcal{Q}}^{4 \mathcal{N}}$ we need to define a quadratic numerical character $\chi_{\mathcal{M}}$ defined modulo $4 \mathcal{M}$, where $\mathcal{M}$ is any integral ideal in the principal complex. For $\mathcal{M}$ in the principal complex, take $a \in \mathcal{O}$ such that $(a, 4 \mathcal{M})=1$; choose $\omega \gg 0$ such that $\omega \mathcal{M}^{-1}=\mathcal{I}^{2}$ for some integral ideal $\mathcal{I}$ with $(\omega, a)=1$. Also choose $\phi_{a} \in \widehat{G}$ to be a fixed representative of $\widehat{G} / H^{\perp}$ such that $\phi_{a}(u)=(u \mid a)$ for all $u \in \mathcal{U}^{+}$. (Notice that the quadratic residue symbol $(* \mid a)$ is a character on $\mathcal{U}^{+} / \mathcal{U}^{2}$, so exactly one of our fixed representatives of $\widehat{G} / H^{\perp}$ extends $(* \mid a)$.) Define $\chi_{\mathcal{M}}(a)=\bar{\phi}_{a}(\omega)(\omega \mid a)$. To show this is well-defined, suppose $\omega^{\prime} \gg 0$ with $\omega^{\prime} \mathcal{M}^{-1}=\mathcal{J}^{2}, \mathcal{J} \subseteq \mathcal{O}$, and $\left(\omega^{\prime}, a\right)=1$. Thus $\mathcal{I}^{2}$ and $\mathcal{J}^{2}$ must be in the same strict ideal class, and hence $\mathcal{I}$ and $\mathcal{J}$ are in the same nonstrict ideal class (recall that $\mathcal{I}_{1}, \ldots, \mathcal{I}_{h^{\prime}}$ represent all the distinct nonstrict classes and $\mathcal{I}_{1}^{2}, \ldots, \mathcal{I}_{h^{\prime}}^{2}$ lie in distinct strict ideal classes). This means that $\omega^{\prime} \omega^{-1} \mathcal{O}=\beta^{2} \mathcal{O}$ for some $\beta \in K^{\times}$, so $\omega^{\prime}=\beta^{2} \omega v$ for some $v \in \mathcal{U}^{+}$. Since $\phi$ and $(* \mid a)$ are quadratic characters and $\phi_{a}(v)=(v \mid a)$, we have

$$
\bar{\phi}_{a}\left(\omega^{\prime}\right)\left(\omega^{\prime} \mid a\right)=\bar{\phi}_{a}(\omega v)(\omega v \mid a)=\bar{\phi}_{a}(\omega)(\omega \mid a) \text {. }
$$

Therefore $\chi_{\mathcal{M}}$ is well-defined. To show $\chi_{\mathcal{M}}$ is a character modulo $4 \mathcal{M}$, take $a$ as above, and choose $q \in \mathcal{M}$. Now, using Dirichlet's theorem on primes, we can choose $\omega_{1}, \omega_{2} \gg 0$ such that $\omega_{i} \mathcal{M}^{-1}=\mathcal{P}_{i}$ where $\mathcal{P}_{i}$ is prime and $\left(\omega_{i}, a\right)=1=\left(\omega_{i}, a+4 q\right)$; then we have $q=\alpha_{1} \omega_{1}+\alpha_{2} \omega_{2}$ for some $\alpha_{i} \in \mathcal{O}$ and $\phi_{a}=\phi_{a+4 \beta}$ for any $\beta \in \mathcal{O}$ since $(u \mid a)=(u \mid a+4 \beta)$ for any $u \in \mathcal{U}^{+}$(note that Theorem 3.7 of [21] implies that $(u \mid *)$ is a character modulo $4 \mathcal{O})$. Noting that $\left(\omega_{i} \mid *\right)$ is a character modulo $4 \omega_{i} \mathcal{M}$ and setting $a^{\prime}=a+4 \alpha_{1} \omega_{1}$ and $a^{\prime \prime}=a^{\prime}+4 \alpha_{2} \omega_{2}=a+4 q$, we see that

$$
\begin{aligned}
\chi_{\mathcal{M}}(a) & =\bar{\phi}_{a}\left(\omega_{1}\right)\left(\omega_{1} \mid a\right)=\bar{\phi}_{a^{\prime}}\left(\omega_{1}\right)\left(\omega_{1} \mid a^{\prime}\right)=\chi_{\mathcal{M}}\left(a^{\prime}\right) \\
& =\bar{\phi}_{a^{\prime}}\left(\omega_{2}\right)\left(\omega_{2} \mid a^{\prime}\right)=\bar{\phi}_{a^{\prime \prime}}\left(\omega_{2}\right)\left(\omega_{2} \mid a^{\prime \prime}\right)=\chi_{\mathcal{M}}(a+4 q) .
\end{aligned}
$$


Hence $\chi_{\mathcal{M}}$ is a character modulo $4 \mathcal{M}$.

With $\mathcal{M}$ in the principal complex we also define a character $\chi_{\mathcal{M}}^{*}$ on ideals relatively prime to $4 \mathcal{M}$ and a Hecke character $\widetilde{\chi_{\mathcal{M}}}$ as follows. For $\mathcal{P}$ a prime ideal not dividing $4 \mathcal{M}$, choose $\omega \gg 0$ such that $\omega \mathcal{M}^{-1}=\mathcal{I}^{2}$ for some integral ideal $\mathcal{I}$ such that $(\omega, \mathcal{P})=1$, and choose $\phi_{\mathcal{P}}$ to be the fixed representative of $\widehat{G} / H^{\perp}$ such that $\phi_{\mathcal{P}}(u)=$ $(u \mid \mathcal{P})$ for all $u \in \mathcal{U}^{+}$. Set

$$
\chi_{\mathcal{M}}^{*}(\mathcal{P})=\bar{\phi}_{\mathcal{P}}(\omega)(\omega \mid \mathcal{P}) ;
$$

extend $\chi_{\mathcal{M}}^{*}$ multiplicatively to all ideals relatively prime to $4 \mathcal{M}$. Set $\widetilde{\chi_{\mathcal{M}}}$ equal to the Hecke character defined by $\widetilde{\chi_{\mathcal{M}}}(\widetilde{s})=\chi_{\mathcal{M}}(\widetilde{s}) \chi_{\mathcal{M}}^{*}(\widetilde{s} \mathcal{O})$ where $\widetilde{s}$ is an idele of $K$ relatively prime to $4 \mathcal{M}$ and $\chi_{\mathcal{M}}(\widetilde{s})$ is the numerical character $\chi_{\mathcal{M}}$ evaluated on the $4 \mathcal{M}$-part of $\widetilde{s}$.

Now, suppose $\mathcal{Q}$ is a prime ideal dividing $\mathcal{N}$ such that $\psi \chi_{\mathcal{Q}}^{\alpha}$ is defined modulo $4 \mathcal{N} \mathcal{Q}^{-\alpha}$ where $\alpha=\operatorname{ord}_{\mathcal{Q}} 4 \mathcal{N}$. If $\mathcal{Q}$ is not in the principal complex, we set $W_{\mathcal{Q}}=W_{\mathcal{Q}}^{4 \mathcal{N}}=0$. So suppose otherwise; for every index $\lambda$, choose an index $\mu$ and $\rho \gg 0$ such that $\mathcal{Q}^{\alpha} \mathcal{I}_{\lambda}^{2}=\rho \mathcal{I}_{\mu}^{2}$. Then for $F=\left(\ldots, f_{\lambda}, \ldots\right) \in \mathcal{M}_{k / 2}(4 \mathcal{N}, \Psi)$ set the $\mu$-component of $F\left|W_{\mathcal{Q}}=F\right| W_{\mathcal{Q}}^{4 \mathcal{N}}$ equal to

$$
\left(\overline{\psi \chi_{\mathcal{Q}}^{\alpha}}\right)_{\mathcal{Q}}(b) \frac{1}{\left[\mathcal{U}^{+}: \mathcal{U}^{2}\right]} \sum_{\phi, u} \bar{\phi}(\rho u) f_{\lambda}\left|\left[\left(\begin{array}{cc}
1 & 0 \\
0 & (\rho u)
\end{array}\right), N(\rho u)^{\frac{1}{4}}\right]\right| C^{*}
$$

where $\left(\psi \chi_{\mathcal{Q}}^{\alpha}\right)_{\mathcal{Q}}$ denotes the $\mathcal{Q}$-part of the character of $\psi \chi_{\mathcal{Q}}^{\alpha}, \phi$ and $u$ vary over $\widehat{G} / H^{\perp}$ and $\mathcal{U}^{+} / \mathcal{U}^{2}$ (respectively), and where

$$
C=\left(\begin{array}{ll}
a & b \\
c & d
\end{array}\right) \in\left(\begin{array}{cc}
\mathcal{Q}^{\alpha} & \mathcal{I}_{\mu}^{-2} \partial^{-1} \\
4 \mathcal{N} \mathcal{Q}^{-\alpha} \mathcal{I}_{\mu}^{2} \partial & \mathcal{O}
\end{array}\right) \cap \Gamma_{1}\left(4 \mathcal{N} \mathcal{Q}^{-\alpha}, \mathcal{I}_{\mu}^{2}\right)
$$

with $\operatorname{det} C=1$. As in the case where $K=\mathbb{Q}, j_{\mathcal{I}_{\mu}}(C, \tau)$ is an automorphy factor for $C$ even when $\mathcal{Q}$ is dyadic, and $W_{\mathcal{Q}}$ is independent of the choice of matrix $C$; as with the operator $T_{\mathcal{Q}}$, the operator $W_{\mathcal{Q}}$ is independent of the choice of $\rho$.

Arguments identical to those used when $K=\mathbb{Q}$ give us

LEMma 4.2. Let $\mathcal{M}, \mathcal{Q}$ be an integral ideals in the principal complex with $\mathcal{Q}$ a prime ideal idividing $\mathcal{N}$. Then we have:

(1) $B_{\mathcal{M}}$ maps $\mathcal{M}_{k / 2}(4 \mathcal{N}, \psi)$ to $\mathcal{M}_{k / 2}\left(4 \mathcal{N} \mathcal{M}, \psi \chi_{\mathcal{M}}\right)$; 
(2) $T_{\mathcal{Q}}$ maps $\mathcal{M}_{k / 2}(4 \mathcal{N}, \psi)$ to $\mathcal{M}_{k / 2}\left(4 \mathcal{N}, \psi \chi_{\mathcal{Q}}\right)$;

(3) If $\psi \chi_{\mathcal{Q}}^{\alpha}$ is defined modulo $4 \mathcal{N Q}^{-\alpha}$ where $\alpha=\operatorname{ord}_{\mathcal{Q}} 4 \mathcal{N}$, then $W_{\mathcal{Q}}$ maps $\mathcal{M}_{k / 2}(4 \mathcal{N}, \psi)$ to $\mathcal{M}_{k / 2}\left(4 \mathcal{N}, \bar{\psi}_{\mathcal{Q}} \psi_{4 \mathcal{N} \mathcal{Q}^{-\alpha}} \chi_{\mathcal{Q}}^{\alpha}\right)$.

Furthermore, the techniques developed in $[\mathbf{2 1}]$ and $[\mathbf{2 3}]$ show that for $\mathcal{P}$ a prime ideal not dividing $4 \mathcal{N} \mathcal{Q}, F=\left(\ldots, f_{\lambda}, \ldots\right) \in$ $\mathcal{M}_{k / 2}(4 \mathcal{N}, \Psi)$, and $u, \gamma$ and $\eta$ as in the definition of $B_{\mathcal{Q}}$ with $(\gamma, \mathcal{P})$ $=1$,

$$
\begin{aligned}
f_{\lambda}\left|\left[\left(\begin{array}{rr}
\gamma u & 0 \\
0 & 1
\end{array}\right), N(\gamma u)^{-\frac{1}{4}}\right]\right| S_{\mathcal{P}} & \\
& =\left(\gamma u \alpha^{2} \mid d^{\prime} \mathcal{P}\right) f_{\lambda}\left|S_{\mathcal{P}}\right|\left[\left(\begin{array}{cc}
\gamma u & 0 \\
0 & 1
\end{array}\right), N(\gamma u)^{-\frac{1}{4}}\right]
\end{aligned}
$$

where $d^{\prime} \in \mathcal{O}, d^{\prime} \equiv 1(\bmod 4 \mathcal{O})$, and $\alpha \in K^{\times}$such that $\left(\gamma u \alpha^{2}, d^{\prime} \mathcal{P}\right)$ $=1$. (Here $d^{\prime}$ is the lower right entry of a matrix used to define $S_{\mathcal{P}}$ on the $\lambda$-component of $F$.) Since $d^{\prime}$ is (in Hecke's terminology) primary and $\gamma u \alpha^{2} \gg 0$, Hecke's Law of Quadratic Reciprocity (see [3]) shows that $\left(\gamma u \alpha^{2} \mid d^{\prime}\right)=1$. Thus taking $\phi_{\mathcal{P}} \in \widehat{G} / H^{\perp}$ such that $\phi_{\mathcal{P}}(u)=(u \mid \mathcal{P})$ for all $u \in \mathcal{U}^{+}$, we have

$$
\begin{aligned}
f_{\lambda}\left|B_{\mathcal{Q}}\right| S_{\mathcal{P}}= & \frac{N(\mathcal{Q})^{-k / 4}}{\left[\mathcal{U}^{+}: \mathcal{U}^{2}\right]} \sum_{\phi, u} \bar{\phi}(\gamma u) \cdot \\
& f_{\lambda}\left|\left[\left(\begin{array}{cc}
\gamma u & 0 \\
0 & 1
\end{array}\right), N(\gamma u)^{-1 / 4}\right]\right| S_{\mathcal{P}} \\
= & \frac{N(\mathcal{Q})^{-k / 4}}{\left[\mathcal{U}^{+}: \mathcal{U}^{2}\right]} \sum_{\phi, u} \bar{\phi}(\gamma u)\left(\gamma u \alpha^{2} \mid \mathcal{P}\right) . \\
& f_{\lambda}\left|S_{\mathcal{P}}\right|\left[\left(\begin{array}{cc}
\gamma u & 0 \\
0 & 1
\end{array}\right), N(\gamma u)^{-1 / 4}\right] \\
= & \frac{N(\mathcal{Q})^{-k / 4}}{\left[\mathcal{U}^{+}: \mathcal{U}^{2}\right]} \sum_{\phi, u} \bar{\phi} \phi_{\mathcal{P}}(\gamma u)\left(\bar{\phi}_{\mathcal{P}}(\gamma u)(\gamma u \mid \mathcal{P})\right) . \\
& f_{\lambda}\left|S_{\mathcal{P}}\right|\left[\left(\begin{array}{cc}
\gamma u & 0 \\
0 & 1
\end{array}\right), N(\gamma u)^{-1 / 4}\right]
\end{aligned}
$$

and since $\gamma \mathcal{Q}^{-1}=\mathcal{I}^{2}$ with $(\gamma, \mathcal{P})=1$ and $\phi \phi_{\mathcal{P}}$ varies over $\widehat{G} / H^{\perp}$ as $\phi$ does, 


$$
\begin{aligned}
& =\chi_{\mathcal{Q}}^{*}(\mathcal{P}) f_{\lambda}\left|S_{\mathcal{P}}\right| B_{\mathcal{Q}} \\
& =\chi_{\mathcal{Q}}^{*} \Psi^{*}(\mathcal{P}) f_{\lambda} \mid B_{\mathcal{Q}}
\end{aligned}
$$

This together with similar arguments give us

LEMMA 4.3. Let $\mathcal{M}, \mathcal{Q}$ be an integral ideals in the principal complex with $\mathcal{Q}$ a prime ideal dividing $\mathcal{N}$. Then we have:

(1) $B_{\mathcal{M}}$ maps $\mathcal{M}_{k / 2}(4 \mathcal{N}, \Psi)$ to $\mathcal{M}_{k / 2}\left(4 \mathcal{N} \mathcal{M}, \Psi \widetilde{\chi_{\mathcal{M}}}\right)$;

(2) $T_{\mathcal{Q}}$ maps $\mathcal{M}_{k / 2}(4 \mathcal{N}, \Psi)$ to $\mathcal{M}_{k / 2}\left(4 \mathcal{N}, \Psi \widetilde{\chi_{\mathcal{Q}}}\right)$;

(3) If $\psi \chi_{\mathcal{Q}}^{\alpha}$ is defined modulo $4 \mathcal{N} \mathcal{Q}^{-\alpha}$ where $\alpha=\operatorname{ord}_{\mathcal{Q}} 4 \mathcal{N}=1$, then $T_{\mathcal{Q}}+N(\mathcal{Q})^{\frac{k}{4}-1} W_{\mathcal{Q}}$ maps $\mathcal{M}_{k / 2}(4 \mathcal{N}, \Psi)$ to $\mathcal{M}_{k / 2}\left(4 \mathcal{N} \mathcal{Q}^{-1}, \Psi \widetilde{\chi_{\mathcal{Q}}}\right)$.

We now examine the effect of the operators $T_{\mathcal{Q}}$ and $B_{\mathcal{Q}}$ on the Fourier coefficients of $F \in \mathcal{M}_{k / 2}(4 \mathcal{N}, \Psi)$ in the case that $\mathcal{Q}$ is an ideal in the principal complex. Writing $f_{\lambda}(\tau)=\sum_{\zeta \in \mathcal{I}_{\lambda}^{2}} a_{\lambda}(\zeta) \mathrm{e}(2 \zeta \tau)$ where $F=\left(\ldots, f_{\lambda}, \ldots\right)$ and taking $\eta$ and $\gamma \gg 0$ as in the definition of $W_{\mathcal{Q}}$, we see that the $\eta$-component of $F \mid B_{\mathcal{Q}}(\tau)$ is

$$
\begin{aligned}
& \frac{N(\mathcal{Q})^{-k / 4}}{\left[\mathcal{U}^{+}: \mathcal{U}^{2}\right]} \sum_{\phi, u} \bar{\phi}(\gamma u) f_{\lambda} \mid\left[\left(\begin{array}{cc}
\gamma u & 0 \\
0 & 1
\end{array}\right), N(\gamma u)^{-1 / 4}\right](\tau) \\
& \quad=\frac{N\left(\mathcal{I}_{\eta} \mathcal{I}_{\lambda}^{-1}\right)^{k / 2}}{\left[\mathcal{U}^{+}: \mathcal{U}^{2}\right]} \sum_{\phi, u} \sum_{\zeta \in \mathcal{Q I}_{\eta}^{2}} a_{\lambda}\left(\zeta \gamma^{-1} u^{-1}\right) \bar{\phi}(\gamma u) \mathrm{e}(2 \zeta \tau) .
\end{aligned}
$$

Thus for $\xi \gg 0$ and $\mathcal{M}=\xi \mathcal{I}_{\lambda}^{-2} \subseteq \mathcal{O}$,

$$
\begin{aligned}
& \mathbf{a}\left(\mathcal{Q M}, \alpha ; F \mid B_{\mathcal{Q}}\right) \\
& \quad=\frac{N\left(\mathcal{I}_{\lambda}\right)^{-k / 2}}{\left[\mathcal{U}^{+}: \mathcal{U}^{2}\right]^{2}} \sum_{u, v \in \mathcal{U}^{+} / \mathcal{U}^{2}} \sum_{\phi \in \widehat{G} / H^{\perp}} \bar{\alpha}(\xi \gamma v) \bar{\phi}(\gamma u) a_{\lambda}\left(\xi v u^{-1}\right)
\end{aligned}
$$

and replacing $v$ by $u v$,

$$
=\frac{N\left(\mathcal{I}_{\lambda}\right)^{-k / 2}}{\left[\mathcal{U}^{+}: \mathcal{U}^{2}\right]^{2}} \sum_{u, v \in \mathcal{U}^{+} / \mathcal{U}^{2}} \sum_{\phi \in \widehat{G} / H^{\perp}} \bar{\alpha}(\xi \gamma v u) \bar{\phi}(\gamma u) a_{\lambda}(\xi v) .
$$

For fixed $v$,

$$
\sum_{\phi, u} \bar{\alpha}(\xi \gamma v u) \bar{\phi}(\gamma u)=\bar{\alpha}(\xi v) \sum_{\phi, u} \overline{\alpha \phi}(\gamma u)=\left[\mathcal{U}^{+}: \mathcal{U}^{2}\right] \bar{\alpha}(\xi v)
$$


since $\sum_{u} \overline{\alpha \phi}(\gamma u)=\overline{\alpha \phi}(\gamma) \sum_{u} \overline{\alpha \phi}(u)=0$ unless $\alpha \phi=1$. Thus

$$
\mathbf{a}\left(\mathcal{Q} \mathcal{M}, \alpha ; F \mid B_{\mathcal{Q}}\right)=\frac{N\left(\mathcal{I}_{\lambda}\right)^{-k / 2}}{\left[\mathcal{U}^{+}: \mathcal{U}^{2}\right]} \sum_{v} \bar{\alpha}(\xi v) a_{\lambda}(\xi v)=\mathbf{a}(\mathcal{M}, \alpha ; F) .
$$

Now suppose that $\mathcal{Q}$ is a prime ideal in the principal complex such that $\mathcal{Q}$ divides $\mathcal{N}$. Then taking $\rho \gg 0$ and $\mu$ as in the definition of $T_{\mathcal{Q}}$, we see that the $\mu$-component of $F \mid T_{\mathcal{Q}}(\tau)$ is

$$
\begin{aligned}
& \frac{N(\mathcal{Q})^{\frac{k}{4}-1}}{\left[\mathcal{U}^{+}: \mathcal{U}^{2}\right]} \sum_{\phi, u} \bar{\phi}(\rho u) \cdot \\
& \qquad \sum_{\zeta \in \mathcal{I}_{\lambda}^{2}} a_{\lambda}(\zeta) \mathrm{e}(2 \zeta \tau)\left|\left[\left(\begin{array}{cc}
1 & 0 \\
0 & \rho u
\end{array}\right), N(\rho u)^{\frac{1}{4}}\right]\right| \sum_{\beta}\left[\left(\begin{array}{ll}
1 & \beta \\
0 & 1
\end{array}\right), 1\right]
\end{aligned}
$$

(where $\beta$ runs over $\mathcal{I}_{\mu}^{-2} \partial^{-1} / \mathcal{Q I}_{\mu}^{-2} \partial^{-1}$ )

$$
\begin{aligned}
& =\frac{N\left(\mathcal{I}_{\mu} \mathcal{I}_{\lambda}^{-1}\right)^{k / 2}}{\left[\mathcal{U}^{+}: \mathcal{U}^{2}\right]} \sum_{\phi, u} \sum_{\zeta \in \mathcal{Q I}_{\lambda}^{2}} \bar{\phi}(\rho u) a_{\lambda}(\zeta) \mathrm{e}\left(2 \zeta(\rho u)^{-1} \tau\right) \\
& =\frac{N\left(\mathcal{I}_{\mu} \mathcal{I}_{\lambda}^{-1}\right)^{k / 2}}{\left[\mathcal{U}^{+}: \mathcal{U}^{2}\right]} \sum_{\phi, u} \sum_{\zeta \in \mathcal{I}_{\mu}^{2}} \bar{\phi}(\rho u) a_{\lambda}(\zeta \rho u) \mathrm{e}(2 \zeta \tau) .
\end{aligned}
$$

Thus for $\mathcal{M}=\xi \mathcal{I}_{\mu}^{-2}, \xi \gg 0$,

$$
\begin{aligned}
& \mathbf{a}\left(\mathcal{M}, \alpha ; F \mid T_{\mathcal{Q}}\right) \\
& =\frac{N\left(\mathcal{I}_{\lambda}\right)^{-k / 2}}{\left[\mathcal{U}^{+}: \mathcal{U}^{2}\right]^{2}} \sum_{u, v \in \mathcal{U}^{+} / \mathcal{U}^{2}} \sum_{\phi \in \widehat{G} / H^{\perp}} \bar{\phi}(\rho u) \bar{\alpha}(\xi v) a_{\lambda}(\xi \rho u v)
\end{aligned}
$$

which replacing $v$ by $v u^{-1}$ becomes

$$
\frac{N\left(\mathcal{I}_{\lambda}\right)^{-k / 2}}{\left[\mathcal{U}^{+}: \mathcal{U}^{2}\right]^{2}} \sum_{u, v \in \mathcal{U}^{+} / \mathcal{U}^{2}} \sum_{\phi \in \widehat{G} / H^{\perp}} \bar{\alpha}(\xi \rho v) \bar{\phi} \alpha(\rho u) a_{\lambda}(\xi \rho v)
$$

Since $\sum_{u} \bar{\phi} \alpha(u)=0$ except when $\bar{\phi} \alpha=1$, we get

$$
\begin{aligned}
\mathbf{a}\left(\mathcal{M}, \alpha ; F \mid T_{\mathcal{Q}}\right) & =\frac{N\left(\mathcal{I}_{\lambda}\right)^{-k / 2}}{\left[\mathcal{U}^{+}: \mathcal{U}^{2}\right]} \sum_{v \in \mathcal{U}^{+} / \mathcal{U}^{2}} \bar{\alpha}(\xi \rho v) a_{\lambda}(\xi \rho v) \\
& =\mathbf{a}(\mathcal{Q M}, \alpha ; F) .
\end{aligned}
$$


Define $S_{k / 2}(4 \mathcal{N}, \Psi)$ to be the subspace of $\mathcal{M}_{k / 2}(4 \mathcal{N}, \Psi)$ consisting of cusp forms and $S_{k / 2}^{-}(4 \mathcal{N}, \Psi)$ the subspace of $S_{k / 2}(4 \mathcal{N}, \Psi)$ spanned by forms of lower levels (i.e. forms of some level $4 \mathcal{N}^{\prime}$ where $\mathcal{N}^{\prime} \supset$ $\mathcal{N}$ ) and shifts (via $B_{\mathcal{Q}}$ ) of such forms. With the Petersson inner product on $S_{k / 2}(4 \mathcal{N}, \Psi)$ defined as in formula (3.3) of [15], we set $S_{k / 2}^{+}(4 \mathcal{N}, \Psi)$ equal to the orthogonal complement of $S_{k / 2}^{-}(4 \mathcal{N}, \Psi)$. Now arguments identical to those used in the case where $K=\mathbb{Q}$ allow us to extend Propositions 3.1-3.7 and Theorem 3.8 to the case where $K$ is any totally real number field. Thus we get

THEOREM 4.4. Suppose $F_{1}, F_{2} \in S_{k / 2}^{+}(4 \mathcal{N}, \Psi)$ and suppose that

(1) for almost all prime ideals $\mathcal{P} \nmid 4 \mathcal{N}, F_{1} \mid T_{\mathcal{P}^{2}}=\lambda_{\mathcal{P}} F_{1}$ and $F_{2} \mid T_{\mathcal{P}^{2}}=\lambda_{\mathcal{P}} F_{2} ;$ and

(2) for almost all squarefree integral ideals $\mathcal{M}, \mathbf{a}\left(\mathcal{M}, \phi ; F_{1}\right)=$ $\mathbf{b}\left(\mathcal{M}, \phi ; F_{2}\right)$ for all $\phi \in \widehat{G} / H^{\perp}$.

Then $F_{1}=F_{2}$.

\section{REFERENCES}

[1] B. Cipra, On the Niwa-Shintani Theta-kernel lifting of modular forms, Nagoya Math. J., 91 (1983), 49-117.

[2] Y. Flicker, Automorphic forms on covering groups of GL(2), Invent. Math., 57 (1980), 119-182.

[3] E. Hecke, Lectures on the Theory of Algebraic Numbers, Springer-Verlag, Berlin Heidelberg New York, 1981.

[4] N. Koblitz, Introduction to Elliptic Curves and Modular Forms, SpringerVerlag, New York, 1984.

[5] W. Kohnen, Modular forms of half-integral weight on $\Gamma_{0}(4)$, Math. Ann., 248 (1980), 249-266.

[6] - Newforms of half-integral weight, J. Reine Angew. Math., 333 (1982), 32-72.

[7] W. Li, Newforms and functional equations, Math. Ann., 212 (1975), 285-315.

[8] M. Manickam, B. Ramakrishnan and T. Vasudevan, On the theory of newforms of half-integral weight, J. Number Theory, 34 (1990), 210-224.

[9] S. Niwa, Modular forms of half-integral weight and the integral of certain Theta-functions, Nagoya Math. J., 56 (1974), 147-161.

[10] J.-P. Serre and H. Stark, Modular Forms of Weight 1/2, Lecture Notes in Math., vol 627, Springer-Verlag, Berlin and New York, (1977), 27-67.

[11] T. Shemanske, Cuspidal newforms and character twists, J. Reine Angew. Math., 328 (1981), 58-71. 
[12] T. Shemanske and L. Walling, Twists of Hilbert modular forms, Trans. Amer. Math. Soc., 338 (1993), 375-403.

[13] G. Shimura, The critical values of certain Zeta functions associated with modular forms of half-integral weight, J. Math. Soc. Japan, 33 (1981), 649-672.

[14] - On modular forms of half-integral weight, Annals of Math., 97 (1973), 440-481.

[15] - On Hilbert modular forms of half-integral weight, Duke Math. J., 55 (1987), 765-838.

[16] M. Ueda, The decomposition of the spaces of cusp forms of half-integral weight and the trace formula of Hecke operators, J. Math. Kyoto U., 28 (1988), 505-555.

[17] - Newforms of half-integral weight and the twisting operators, Proc. Japan Acad., 66 (1990), 173-175.

[18] M. -F. Vignéras, Facteurs Gamma et Équations Fonctionnelles, Lecture Notes in Math., vol 627, Springer-Verlag, Berlin and New York, (1977), 79-103.

[19] - Valeur au Centre de Symetrie des fonctions L associees aux formes modulaires, Seminaire de Theorie de Nombres, Paris, 1979-1980, Progress in Math, vol. 12, Birkauser, Boston, (1981), 331-356.

[20] J. Waldspurger, Sur les Coefficients de Fourier des Formes Modulaires de Poids Demi-Entier, J. Math Pures Appl., 60 (1981), 375-484.

[21] L. Walling, Hecke operators on Theta series attached to lattices of arbitrary rank, Acta Arith., 54 (1990), 213-240.

[22] - On lifting Hecke eigenforms, Trans. Amer. Math. Soc., 328 (1991), 881-896.

[23] - Hecke eigenforms and representation numbers of arbitrary rank lattices, Pacific J. Math., 156 (1992), 371-394.

Received September 9, 1992.

Dartmouth College

HANOVER, NEW HAMPSHIRE 03755

E-mail address: Thomas.Shemanske@dartmouth.edu

AND

UNIVERSITY OF COLORADO

BOULDER, COLORADO 80309

Email address: walling@euclid.Colorado.edu 




\section{PACIFIC JOURNAL OF MATHEMATICS}

Volume $167 \quad$ No. $2 \quad$ February 1995

Existence of shortest directed networks in $\mathbb{R}^{2}$

201

MANUEL AlFARO GARCIA

Hecke characters of singular Drinfel'd modules

215

SUNGHAN BAE

Factorization method for a bimeromorphic morphism

231

Jose PEREZ BLANCO

$L^{p}$ estimates for operators associated to flat curves without the Fourier 243 transform

Anthony Carbery, James Thomas Vance, JR., Stephen

WAINGER, DAVID K. WATSON and JAMES WRIGHT

$S$-integer points on elliptic curves

263

ROBERT HOWARD GROSS and JOSEPH SILVERMAN

On metrics defined by modules

JAMES ALLISTER JENKINS

Conditional Wiener integrals. II

ChUll PARK and DAVID LeE SKOUG

On a Plancherel formula for certain discrete, finitely generated, torsion-free nilpotent groups

CAROLYN PFEFFER JOHNSTON

Desingularizations of some unstable orbit closures

MARK STEPHEN REEDER

Determining multiplicities of half-integral weight newforms

Thomas Richard SHEMANSKE and LyNne WALLING

Generation of integral orthogonal groups over dyadic local fields 